Utah State University

DigitalCommons@USU

\title{
The extent and characteristics of low productivity aspen areas in Minnesota
}

\author{
G.K. Raile
}

J.T. Hahn

Follow this and additional works at: https://digitalcommons.usu.edu/aspen_bib

Part of the Forest Sciences Commons

\section{Recommended Citation}

Raile, G.K. and Hahn, J.T., "The extent and characteristics of low productivity aspen areas in Minnesota" (1982). Aspen Bibliography. Paper 4261.

https://digitalcommons.usu.edu/aspen_bib/4261

This Article is brought to you for free and open access by the Aspen Research at DigitalCommons@USU. It has been accepted for inclusion in Aspen Bibliography by an authorized administrator of DigitalCommons@USU. For more information, please contact

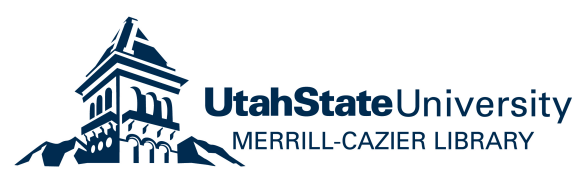




\title{
EXTENT AND CHARACTERISTICS OF LOW PRODUCTIVITY ASPEN AREAS IN MINNESOTA
}

\author{
Gerhard K. Raile, Mensurationist, \\ and Jerold T. Hahn, Principal Mensurationist
}

The aspen type in Minnesota occupies about 5.3 million acres, 39 percent of the State's commercial forest land ${ }^{1}$ Aspen is the State's major commercial species, accounting for 43 percent of all growingstock removals for products in 1976 (Jakes 1980). In recent years the use of aspen has grown even larger because of new waferboard plants in the State. Because of the wide extent of the aspen type and its importance to forest industry, there is much interest in knowing the productive capacity of aspen forest land.

Extensive areas of aspen in Minnesota currently produce little commercial timber, because stands are understocked, overmature, or on low sites. Details about the extent, characteristics, and distribution of these low productivity areas have not been available. Lundgren and Hahn (1978) developed a method for assessing the productivity of aspen areas in Wisconsin. A similar method will be used in this report to provide a basis for comparison between Minnesota and Wisconsin aspen forests. In addition, we developed new equations to evaluate aspen site productivity and potential productivity achieved. This report summarizes the current and potential productivity and general location and distribution of the aspen forest type in Minnesota.

\section{DATA BASE}

The data for this study were taken from 3,398 commercial forest plots measured on non-National Forest land during the 1977 forest survey of Minnesota (Jakes 1980). Additional data included 62 aspen plots on the Superior National Forest (measured

${ }^{1}$ Commercial forest land and other terms used throughout this paper are defined in the Appendix. in 1979) and 62 aspen plots on the Chippewa National Forest (measured in 1980). Each plot consisted of 10 variable radius ( 37.5 basal area factor prism) points spread equally over approximately 1 acre. Plot locations were determined by placing a systematic grid of 1-acre dots over an aerial photo mosaic of each township. The State was divided into four Survey Units (fig. 1) for computing and for presenting the data by smaller, geographically similar sections.

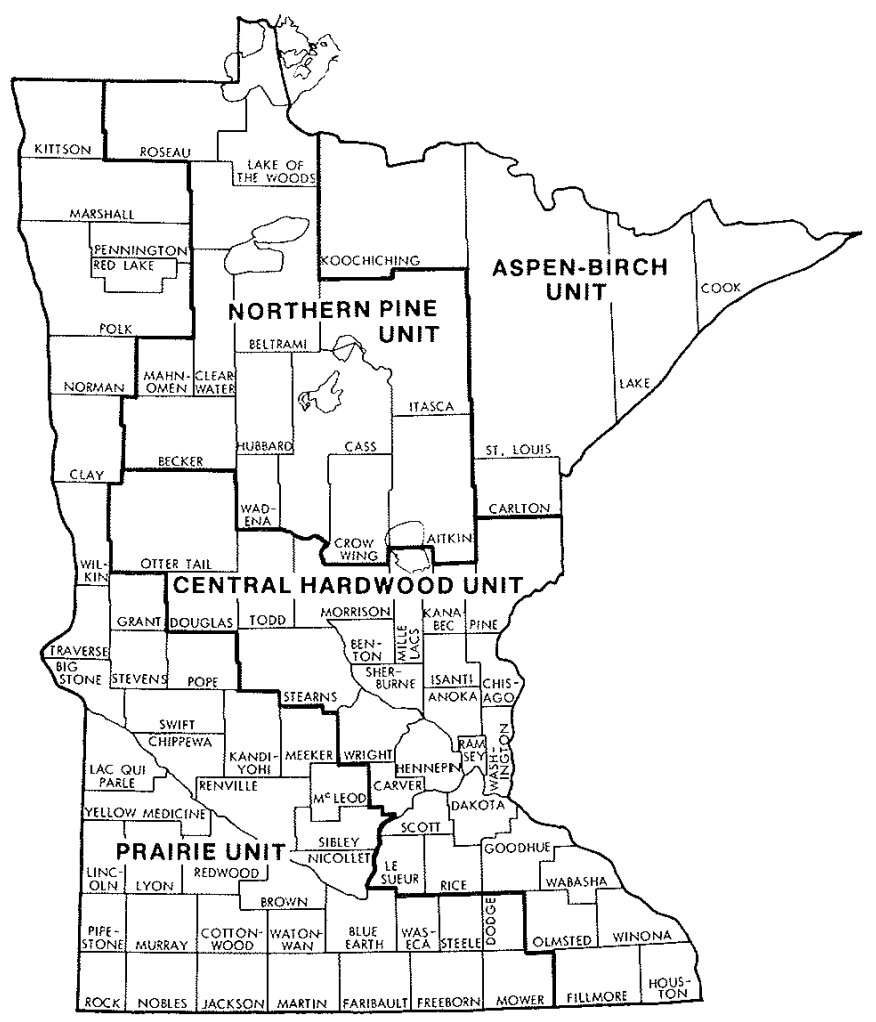

Figure 1.-Survey units for the 1977 Minnesota Survey. 


\section{METHODS Site Productivity}

The first step in the analysis was to determine the site productivity of each aspen inventory plot in Minnesota. Site productivity is expressed in cubic feet of growth per acre per year. The current productivity of a stand varies with its age, but for a given rotation length a stand's productivity can be expressed in terms of the mean annual increment over the rotation. Site productivity typically is expressed as the maximum mean annual increment (MMAI) over the range of possible rotation ages.

Yield tables from Kittredge and Gevorkiantz (1929), reporting total peeled cubic foot volume per acre per year in trees 1.0 inch d.b.h. and larger in well-stocked aspen stands, have been used for decades as the standard for determining aspen site productivity in the Lake States. Lundgren and Hahn (1978) used these yield tables to estimate site productivity for aspen in Wisconsin. They developed an equation relating MMAI in cubic feet per acre per year to site index (S) from the table data in Kittredge and Gevorkiantz (1929), resulting in the following equation:

$$
\text { MMAI }=-97.53+3.5463 \mathrm{~S}-.014286 \mathrm{~S}^{2}
$$

To improve on this estimate of site productivity, we used North Central Forest Experiment Station's Stand and Tree Evaluation and Modeling System (STEMS) (Belcher 1981) to develop an aspen yield function for fully stocked stands. STEMS is a system of computer programs that projects the growth of individual trees in stands. Based on biological principles of forest growth, it offers a flexible, generalized approach to simulating the change of forest trees in stands. STEMS was used to simulate the growth of fully stocked aspen stands from age 5 to 95 over the entire range of commercial aspen site indices (35 to 105). Stand volume and basal area were computed every 5 years. These data were then used to develop the following yield function:

$$
\mathrm{V}=30.6 \mathrm{~S}^{1.111}\left(1-\mathrm{e}^{-.12065 \mathrm{~A})} 5928 \mathrm{~S}^{-1.189}\right.
$$

where

$$
\begin{aligned}
& \mathrm{V}=\text { Volume of the stand (cubic feet/acre) } \\
& \mathrm{S}=\text { site index (feet at } 50 \text { years) } \\
& \mathrm{A}=\text { stand age (years) }
\end{aligned}
$$

Iteration was then used with this equation to compute the MMAI for all the aspen stands in Minnesota. We used both the STEMS MMAI and the MMAI from equation 1 to classify each plot's potential site productivity. Except for the least productive sites, the STEMS MMAI is lower than the MMAI from equation (1).

\section{Potential Achieved}

Next, we developed a method to evaluate each plot's achievement of its potential productivity. For several reasons, we used current basal area per acre rather than growth or volume to compare potential achieved to site productivity. First, the actual current annual increment recorded for each inventory plot cannot be compared directly with the MMAI. Current increment is only the net growth for the current year, whereas the MMAI is the maximum average growth throughout an entire rotation.

Second, although we could have used STEMS to project current stands to the end of rotation, many stands are not of pure aspen or of one age, hindering the determination of rotation age.

Third, using basal area to estimate the percent of potential productivity achieved eliminated extensive volume computations and adjustments. Lundgren and Hahn (1978) showed that if we assume stand height is the same for a given age and site regardless of stand density, then the proportion of potential volume achieved on a plot is identical to the proportion of potential basal area achieved.

Lundgren and Hahn (1978) developed the following equation to estimate the potential basal area per acre by fitting a nonlinear function to the basal areas given by Kittredge and Gevorkiantz (1929) for all trees 1.0 inch d.b.h. and larger in well-stocked aspen stands.

$$
\mathrm{B}=2.385 \mathrm{~S}\left(1-\mathrm{e}^{-0.031268 \mathrm{~A}}\right)
$$

where

$$
\begin{aligned}
\mathrm{B}= & \text { potential basal area per acre for all } \\
& \text { live trees (square feet/acre) } \\
\mathrm{S}= & \text { site index (feet at } 50 \text { years) } \\
\mathrm{A}= & \text { stand age (years) }
\end{aligned}
$$

In addition to using equation 3 to estimate the percent achieved, we developed the following model to estimate potential basal area based on the STEMS simulations:

$$
\mathrm{B}=102.7 \mathrm{~S} \cdot 1019\left(1-\mathrm{e}^{-0486 \mathrm{~A}}\right)^{1.2626}
$$

where $\mathrm{B}, \mathrm{S}$, and $\mathrm{A}$ are defined above. 
To estimate the proportion of potential achieved, the current basal area of the plot was divided by its potential, estimated from equation 3 or 4 . With equation 4, the estimated potential basal area of a 30 year-old stand with a site index of 70 would be 113 square feet per acre. If the actual basal area was 68 square feet per acre, the stand would have achieved 60 percent of its potential.

The present basal area of a stand can be obtained directly from each plot record. But should this basal area include all live trees or only growing-stock trees? The first provides a measure of apparent stocking of all trees in a stand and may be most appropriate for a harvest system such as full-tree chipping of all live trees. The second measures stocking of only those trees considered desirable and acceptable by traditional markets and harvesting systems. We used both measures and made separate sets of tables for each for the equations based on STEMS and the equations based on Kittredge and Gevorkiantz (1929).

\section{RESULTS}

The site productivity and percent of potential achieved were determined for each aspen plot and summarized for the State. Tables 4 through 23 in the Appendix include State and Unit totals for both growing-stock and all live trees based on the equations derived from STEMS. In addition, State totals based on Kittredge and Gevorkiantz (1929) are included (Appendix tables 24-27) to allow comparison of Minnesota's aspen type productivity with that of Wisconsin. The total acres within each age class are the same for all methods of computation, but the distribution of acres among achievement and potential productivity classes differs.

The following is an example of how the tables may be used in evaluating aspen productivity in Minnesota. If we define a low productivity area as one with a potential for growing less than 41 cubic feet per acre per year, then there are 321,700 acres of such "low productivity" stands (Appendix table 4) in the State out of a total of $5,377,200$ acres. Or, low productivity could be considered as all stands with a site productivity less than 61 cubic feet per acre per year and all stands presently achieving less than 51 percent of potential based on growing-stock trees. In this case we could add all acres with a site potential less than 61 cubic feet per acre per year $(321,700+2,314,400=2,636,100$ acres $)$ and those areas with a potential greater than 60 cubic feet per acre per year that are at 50 percent or less of potential $(1,152,800$ acres $)$ to get a total low productivity area in Minnesota of 3,788,900 acres.
Maps showing the location of selected aspen plots in Minnesota were prepared using the Universal Transverse Mercator Grid Coordinates (UTM) for each plot. These maps provide a visual impression of plot locations and help identify areas that have a concentration of special categories of aspen plots. A map showing the location of all 3,522 aspen plots in Minnesota provides a visual standard for comparing all the other maps (fig 2).

Plots with the lowest site productivity (MMAI less than 41 cubic feet per acre per year) are shown in figure 3. Timber production from these stands would be low even if full stocking were achieved. Worth noting is the lack of low site plots in large parts of Lake, Cass, and Itasca Counties. Kittson, Roseau, Marshall and western Beltrami Counties have a higher than average percent of low site plots.

The plots that achieve less than 26 percent of their potential based on growing-stock trees are fairly evenly distributed throughout the aspen type (fig. 4). Aspen production would be greatly increased if these stands were fully stocked.

Some plots with a high site productivity (greater than 80 cubic feet per acre per year) are achieving less than half of their potential based on growing stock trees (fig. 5). These plots would be prime candidates for some form of management. They are concentrated in the north-central part of the State, in and around Itasca County.

A fast way to increase productivity would be to harvest overmature aspen stands that have a high productive potential in order to regenerate an adequately stocked aspen stand. Such stands with age greater than 50 and MMAI greater than 80 are concentrated in Itasca and northern St. Louis Counties (fig. 6).

\section{DISCUSSION}

When compared to previous systems and observed conditions, the STEMS potential basal area outlined in this paper appears to give consistent and reasonable results and provides greater flexibility for evaluation. Many plots closely approach their potential for the entire range of sites and ages. For example, 38 percent of the aspen plots in Minnesota had more than three-quarters of their STEMS potential basal area in all live trees. Seventeen percent of all the aspen plots had more than their potential basal area, if all live trees are included. Even if only growingstock basal area is considered, 9 percent of all the 


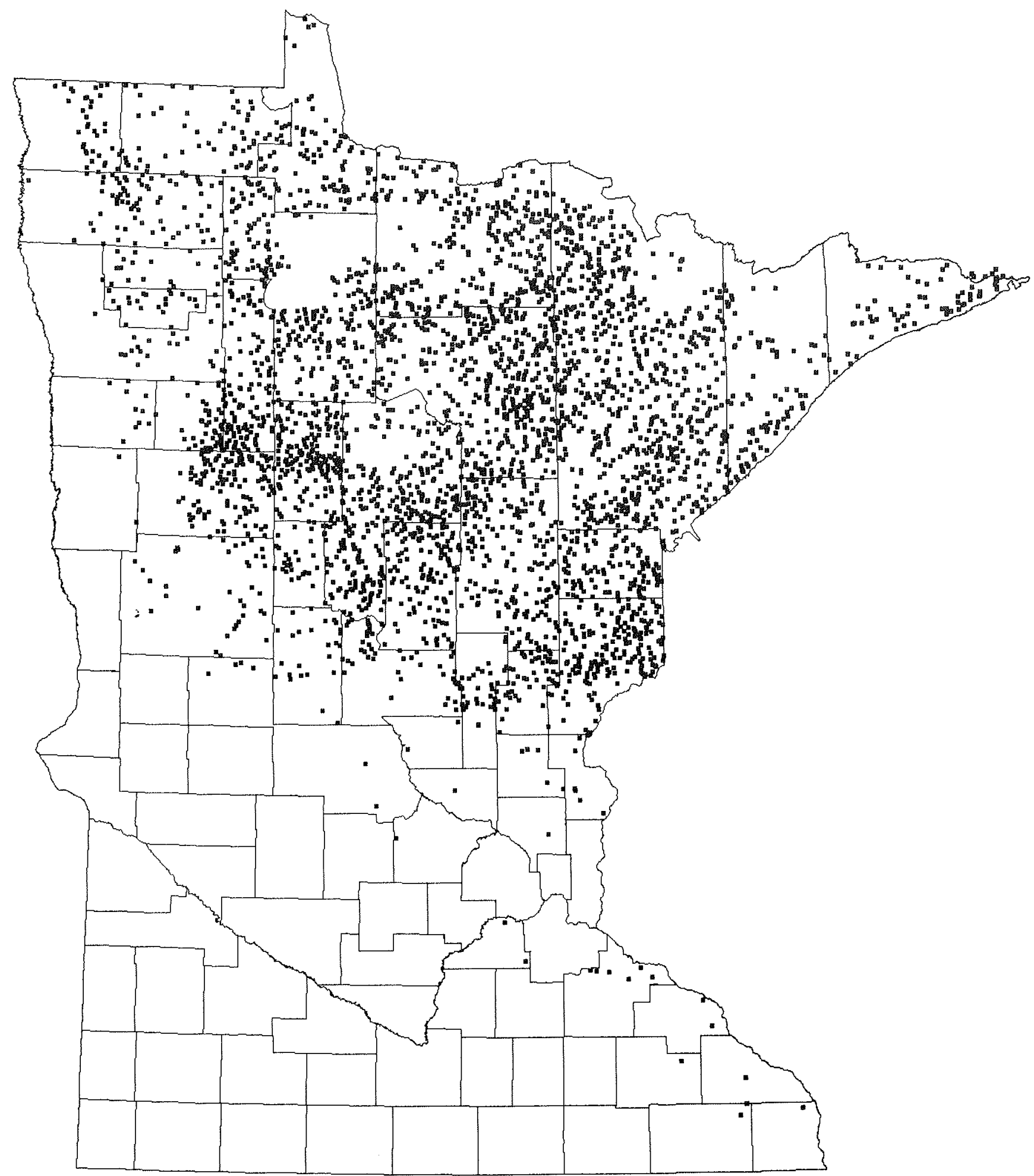

Figure 2.-Location of all inventory plots typed as aspen.

plots exceeded the potential indicated for their specific site and age. This strongly indicates that the potential basal areas used in this report are realistic goals. $^{2}$
${ }^{2}$ The potential basal area equation developed by Lundgren and Hahn (1978) generally gives a lower basal area than the STEMS equation. 


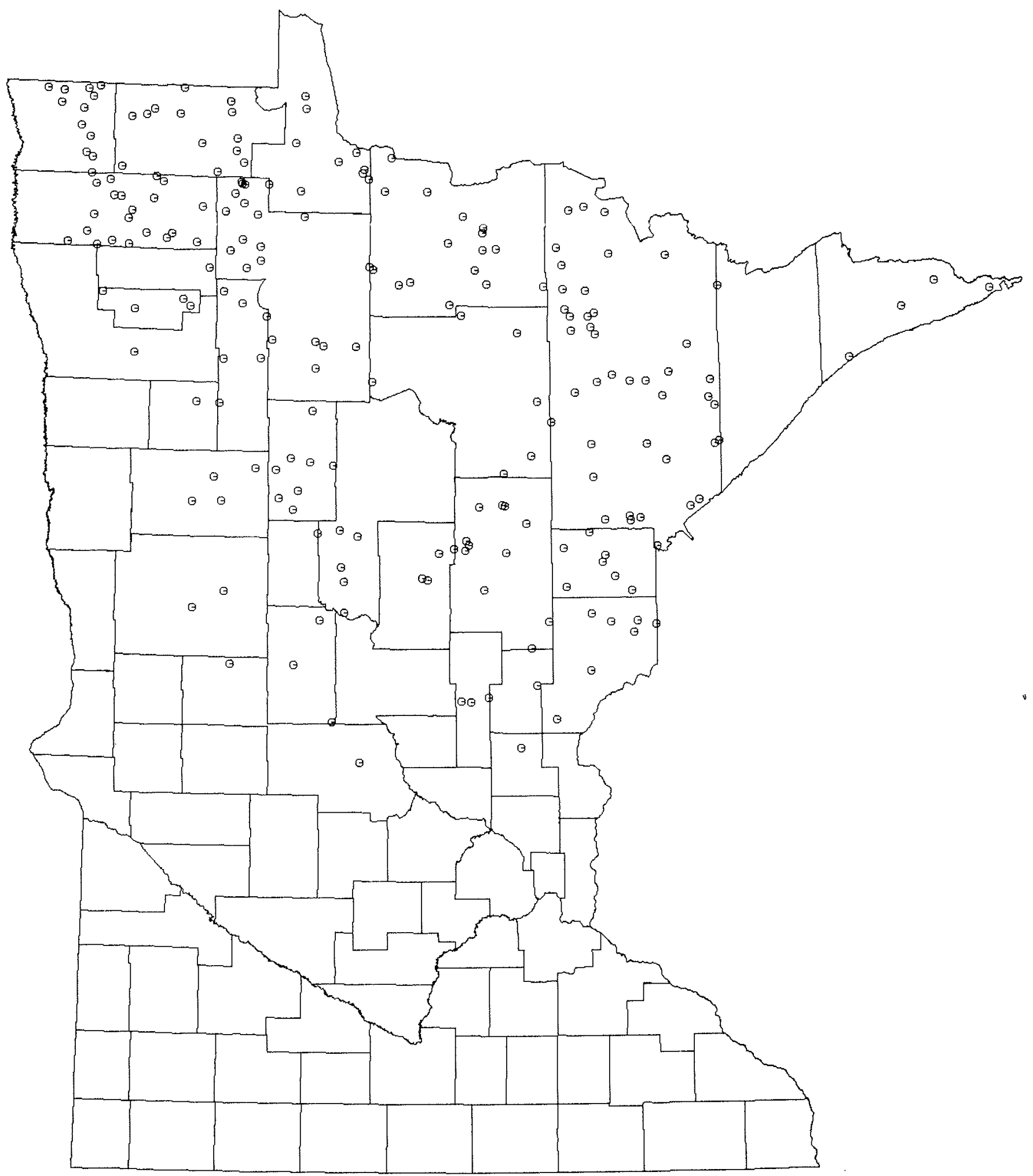

Figure 3.-Location of aspen plots with a site productivity less than 41 cubic feet per acre per year. 


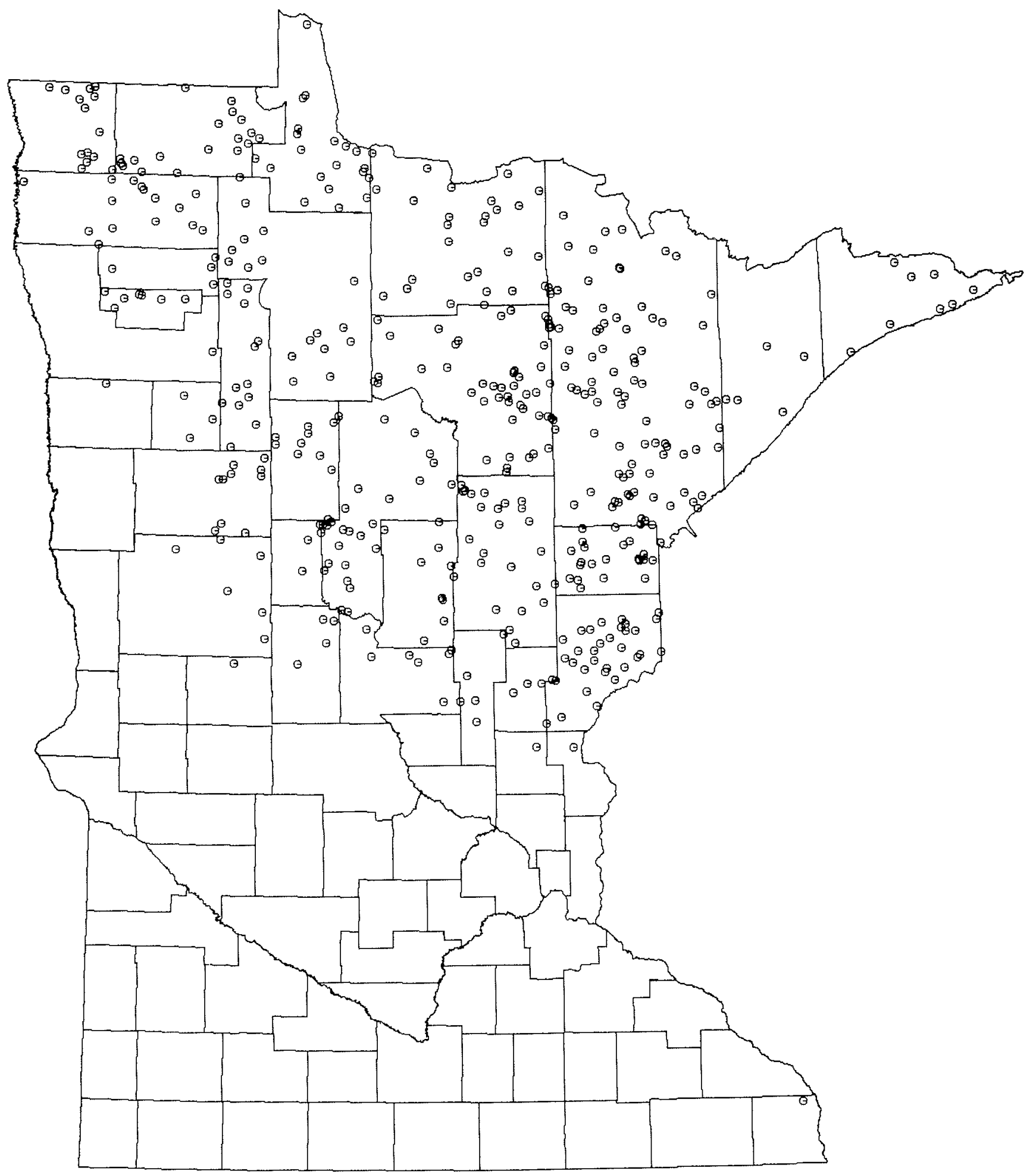

Figure 4.-Location of aspen plots achieving less than 26 percent of STEMS growing stock potential. 


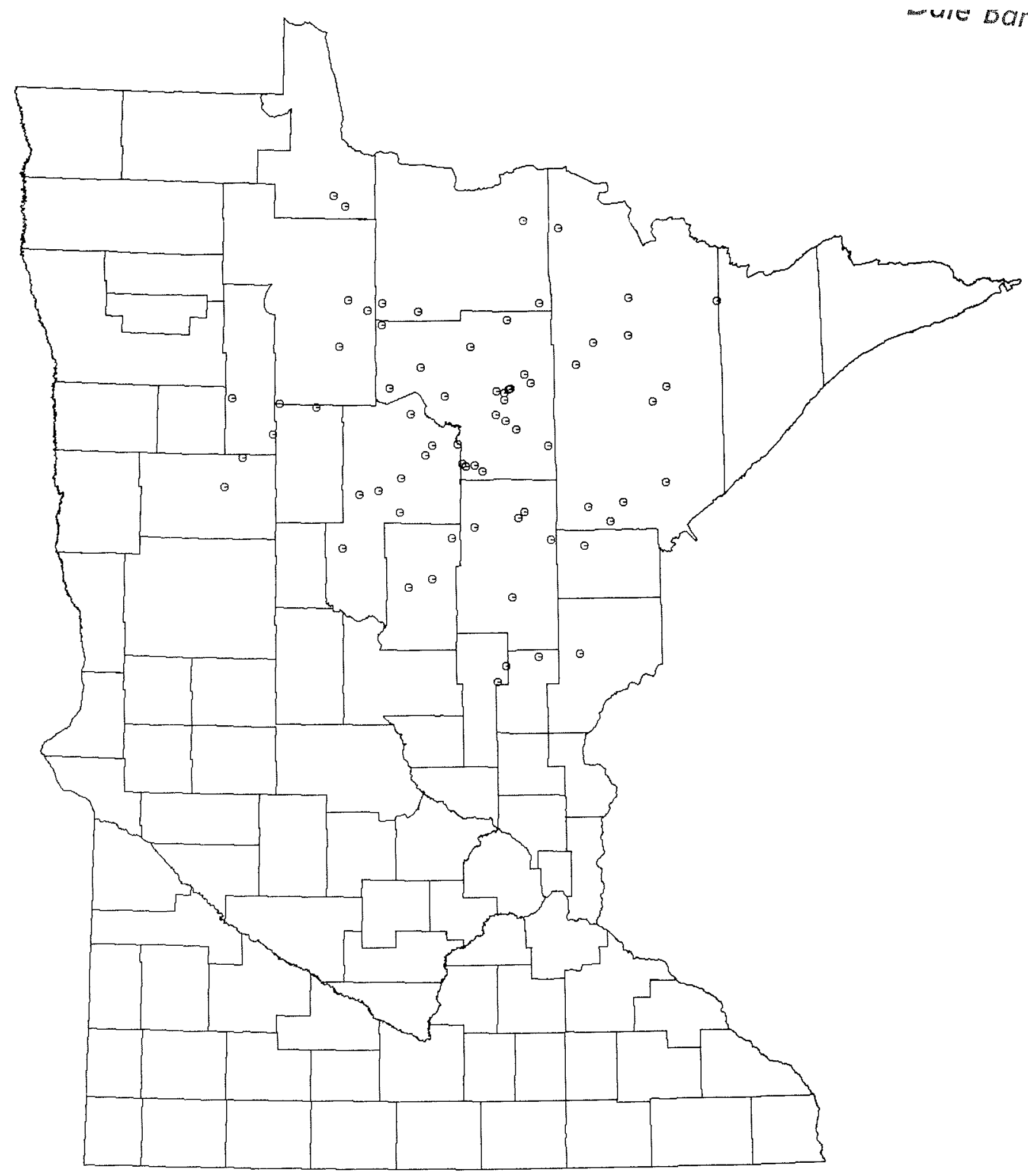

Figure 5.-Location of aspen plots with a site productivity greater than 80 cubic feet per acre per year but achieving less than 51 percent of STEMS growing stock potential. 


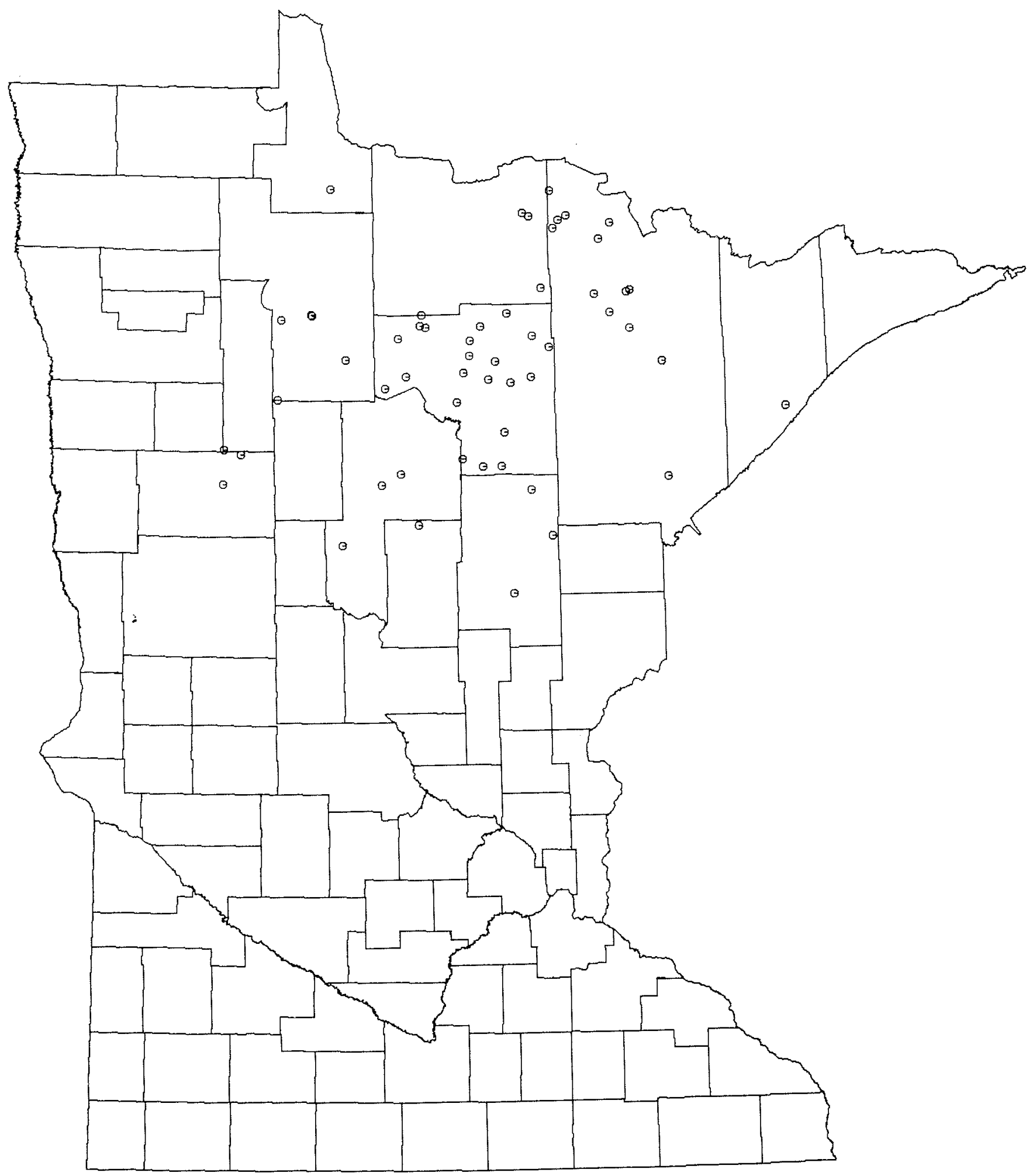

Figure 6.-Location of aspen plots with a site productivity greater than 80 cubic feet per acre per year and a stand age greater than 50 years. 
In Minnesota, 2.6 million acres of aspen (49 percent of the total table 1) currently achieve less than half of their potential, if only growing-stock trees are considered. Even if all live trees are included, 1.5 million acres (29 percent of the aspen type) achieve less than half of their potential. Thus, the potential exists for greatly increasing yield of the aspen type in Minnesota.

If it were desirable and possible to reach full production in all of the aspen type, there could be an annual harvest of 120,100 acres containing a total of 325.6 million cubic feet. ${ }^{3}$ The 1976 aspen growingstock removals for products in Minnesota was 60.3 million cubic feet. This level of removals could be supported by maintaining full stocking on only the best 14.4 percent of aspen sites.

Attaining full stocking on all acres of aspen type is an unrealistic goal (Bruce 1977, Spurr and Vaux 1976). Insect and disease epidemics, windstorms, drought, and many other factors all contribute to understocking and make full stocking on every acre an unattainable goal. However, maintaining full stocking on the best 39 percent of the aspen land would produce as much volume as the entire aspen type currently produces. Research is needed to determine why large areas are understocked and to suggest measures that could be taken to improve stocking.

To achieve full site potential in poorly stocked aspen stands, the existing stand may have to be removed and the site regenerated by appropriate measures. But it may not be desirable to regenerate all sites to aspen - some sites may be better adapted to other species, or the cost of site preparation to ensure full stocking may exceed the benefit.

A summary of acres by physiographic class and site index for all aspen plots may give some clues about the potential for site conversion (tables 14 and 15). Five physiographic classes were used in the Minnesota inventory, ranging from xeric (very dry, droughty sites) through hydric (where growth and number of species are seriously limited by excess water)

Mesic sites constitute 85.4 percent of the aspen type. These sites have the highest average MMAI, 61.4 cubic feet per acre per year; hydric sites have the lowest average MMAI at 51.6 cubic feet per acre

${ }^{3}$ These data are based on the STEMS simulation and assume (1) full area regulation of the aspen type, (2) no thinning, and (3) harvesting at the culmination of mean annual increment.
Table 1.-Potential basal area achieved by aspen commercial forest, by Survey Unit, Minnesota $1977^{1}$ (based on STEMS)

(In percent of commercial aspen area by Survey Unit)

\begin{tabular}{lccccc}
\hline & \multicolumn{4}{c}{ Potential achieved (percent) } & Total \\
\cline { 2 - 5 } Survey unit & $\mathbf{0 - 2 5}$ & $\mathbf{2 6 - 5 0}$ & $\mathbf{5 1 - 7 5}$ & $\mathbf{7 6 +}$ & $\mathbf{0 - 1 0 0 +}$ \\
\hline ALL LIVE TREES & 6 & 24 & 33 & 37 & 100 \\
$\quad$ Aspen-birch & 6 & 19 & 35 & 40 & 100 \\
$\quad$ Northern pine & 7 & 27 & 36 & 30 & 100 \\
Central hardwood & 8 & 24 & 28 & 40 & 100 \\
Prairie & 6 & 22 & 34 & 38 & 100 \\
$\quad$ All units & & & & & \\
GROWING-STOCK TREES & 20 & 42 & 16 & 22 & 100 \\
$\quad$ Aspen-birch & 12 & 34 & 34 & 20 & 100 \\
Northern pine & 16 & 45 & 24 & 15 & 100 \\
Central hardwood & 24 & 31 & 25 & 20 & 100 \\
Prairie & 13 & 36 & 30 & 21 & 100 \\
All units &
\end{tabular}

1 Includes data from Superior National Forest 1979 and Chippewa National Forest 1980.

per year. The distribution of the aspen type by physiographic class is given in table 2. Tables in the Appendix break down the area of the aspen type by site potential, percent achieved, and physiographic class. These data indicate that mesic sites tend to have the highest potential and highest achievement of that potential.

Tables 24 through 27 in the Appendix are directly comparable with the tables produced by Lundgren and Hahn (1978) for Wisconsin. Table 3 shows how the distribution of the aspen type in Minnesota compares with that of Wisconsin. Based on growing-stock trees, Minnesota's aspen type is achieving more of its potential than Wisconsin's. In addition, a larger proportion of Minnesota's aspen sites have a higher productive potential than Wisconsin's.

Table 2.-Physiographic class of aspen commercial forest, Minnesota $1977^{1}$

(In percent of commercial aspen area by Survey Unit)

\begin{tabular}{lrrrrrr}
\hline Survey unit & Xeric & Xeromesic & Mesic & Hydromesic & Hydric & All \\
\hline Aspen-birch & 0.8 & 2.0 & 81.1 & 15.7 & 0.4 & 100.0 \\
Northern pine & .1 & 1.5 & 87.7 & 10.2 & .5 & 100.0 \\
Central hardwood & .5 & .6 & 85.0 & 13.7 & .3 & 100.0 \\
Prairie & - & $\overline{1.5}$ & 86.1 & 3.8 & - & 100.0 \\
All units & .4 & & & 12.3 & .4 & 100.0 \\
\hline
\end{tabular}

IInciudes data from Superior National Forest 1979 and Chippewa National Forest 1980 
Table 3.-Potential basal area achieved by aspen commercial forest by site productivity class, Minnesota 1977 and Wisconsin 1968 (based on Kittredge and Gevorkiantz 1929) ${ }^{1}$

(In percent of total commercial aspen area)

\begin{tabular}{|c|c|c|c|c|c|c|c|c|c|c|}
\hline \multirow{2}{*}{$\begin{array}{l}\text { Site } \\
\text { productivity } \\
\left.\text { ( } \mathrm{t}^{3} / \mathrm{ac} / \mathrm{yr}\right)\end{array}$} & \multicolumn{8}{|c|}{ Potential achieved (percent) } & \multirow{2}{*}{\multicolumn{2}{|c|}{$\begin{array}{c}\text { Total } \\
0-100+\end{array}$}} \\
\hline & \multicolumn{2}{|c|}{$0-25$} & \multicolumn{2}{|c|}{$26-50$} & \multicolumn{2}{|c|}{$51-75$} & \multicolumn{2}{|c|}{$76+$} & & \\
\hline & MN & WI & MN & WI & MN & WI & MN & WI & MN & WI \\
\hline & - & - & & 2 & 2 & 1 & 1 & 1 & 5 & \\
\hline 00 & 2 & 5 & 13 & 10 & 13 & 10 & 8 & 10 & 36 & 35 \\
\hline $61-80$ & 3 & 5 & 11 & 10 & 11 & 10 & 12 & 10 & 38 & 3 \\
\hline $41-60$ & 2 & 5 & 4 & 6 & 4 & 5 & 5 & 5 & 15 & 21 \\
\hline $21-40$ & 1 & 2 & 1 & 2 & 2 & 1 & 2 & 2 & 6 & 6 \\
\hline All sites & 9 & 18 & 31 & 28 & 32 & 26 & 28 & 27 & 100 & 100 \\
\hline
\end{tabular}

1 Includes data from Superior National Forest 1979 and Chippewa National Forest 1980. Wisconsin data is from Lundgren and Hahn (1978).

\section{CONCLUSIONS}

Much forest area classified as aspen type in Minnesota can produce high cubic foot volumes but is currently achieving less than its potential. The 1976 aspen growing-stock removals for products could be grown on the best 14.4 percent of the State's aspen sites. The best aspen sites that are seriously underachieving their potential are located in north central Minnesota in and around Itasca County. Because 55 percent of these sites are in public ownership, research is needed on the possibilities and techniques for reaching full stocking in aspen stands. Whether full productivity is economically, ecologically, or environmentally feasible also must be determined.

\section{LITERATURE CITED}

Belcher, David M. User's Guide to STEMS (Stand and Tree Evaluation and Modeling System). Gen. Tech. Rep. NC-70. St. Paul, MN: U.S. Department of Agriculture, Forest Service, North Central Forest Experiment Station; 1981. 49 p.

Bruce, David. Yield differences between research plots and managed forests. J. For. 75(1): 14-17; 1977.

Jakes, Pamela J. Minnesota Forest Statistics, 1977. Resour. Bull. NC-53. St. Paul, MN: U.S. Department of Agriculture, Forest Service, North Central Forest Experiment Station; 1980. 85 p.

Kittredge, Joseph, Jr.; Gevorkiantz, S. R. Forest possibilities of aspen lands in the Lake States. Tech. Bull. 60. St. Paul, MN: U.S. Department of Agriculture, forest Service, North Central Forest Experiment Station; 1929. $84 \mathrm{p}$.

Lundgren, Allen A.; Hahn, Jerold T. The extent and characteristics of low productivity aspen areas in Wisconsin. Gen. Tech. Rep. NC-45. St. Paul, MN: U.S. Department of Agriculture, Forest Service, North Central Forest Experiment Station; 1978. $24 \mathrm{p}$.

Spurr, Stephen; Vaux, Henry. Timber biological and economic potential. Science 191(4228): 752-756; 1976.

\section{APPENDIX}

\section{DEFINITION OF TERMS}

Acceptable trees. - Growing-stock trees of commercial species that meet specified standards of size and quality but do not qualify as desirable trees.

Basal area.-The area in square feet of the cross section at breast height of a single tree. When the basal area of all trees in a stand are summed, the result is usually expressed as square feet of basal area per acre.

Commercial forest land. - Forest land producing or capable of producing crops of industrial wood and not withdrawn from timber utilization. (Note: Areas qualifying as commerical forest land have the capability of producing in excess of 20 cubic feet per acre per year of annual growth under management. Currently inaccessible and inoperable areas are included, except when the areas involved are small and unlikely to become suitable for production of industrial wood in the foreseeable future.)

Desirable trees.-Growing-stock trees having no serious defects in quality limiting present or prospective use, and of relatively high vigor, and containing no pathogens that may result in death or serious deterioration before rotation age. These are trees that would be favored by forest managers in silvicultural operations.

Forest land.-Land at least 16.7 percent stocked by forest trees of any size, or formerly having had 
such tree cover, and not currently developed for nonforest use. (Note: Stocking is measured by comparison of basal area and/or number of trees, by age or size and spacing with specified standards. The minimum area for classification of forest land is 1 acre. Roadside, streamside, and shelterbelt strips of timber must have a crown width of at least 120 feet to qualify as forest land. Unimproved roads and trails, streams, or other bodies of water or clearings in forest areas shall be classed as forest if less than 120 feet wide.) Also see definitions for land area, commercial forest land, noncommercial forest land, productive-reserved forest land, stocking, unproductive forest land, and water.

Forest type.-A classification of forest land based upon the species forming a plurality of live tree stocking.

Aspen.-Forests in which quaking aspen or bigtooth aspen, singly or in combination, comprise a plurality of the stocking. (Common associates include balsam poplar, balsam fir, and paper birch.)

Growing-stock trees. - Live trees of commercial species qualifying as desirable and acceptable trees. (Note: Excludes rough, rotten, and dead trees.)

Growing-stock volume.- Net volume in cubic feet of growing stock trees 5.0 inches d.b.h. and over, from a 1-foot stump to a minimum 4.0 inch top diameter outside bark of the central stem or to the point where the central stem breaks into limbs. Cubic feet can be converted to cords by multiplying by 79 cubic feet per solid wood cord.

Land area.-A. Bureau of the Census. The area of dry land and land temporarily or partly covered by water, such as marshes, swamps, and river flood plains (omitting tidal flats below mean high tide); streams, sloughs, estuaries, and canals less than one-eighth of a statute mile wide; and lakes, reservoirs, and ponds less than 40 acres in area.

B. Forest Survey. The same as the Bureau of the Census, except minimum width of streams, etc. is 120 feet and minimum size of lakes, etc. is 1 acre.

Live trees.-Growing-stock, rough and rotten trees 1 inch d.b.h. and larger.

National forest land. - Federal land that has been legally designated as National Forest or purchase units, and other land under the administration of the USDA Forest Service.

Noncommercial forest land.-(a) Unproductive forest land and (b) productive-reserved forest land.

Nonforest land.-Land that has never supported forests, and land formerly forested where use for timber management is precluded by development for other uses. (Note: Includes areas used for crops, improved pasture, residential areas, city parks, improved roads of any width and adjoining clearings, powerline clearings of any width, and 1- to 40-acre areas of water classified by the Bureau of the Census as land. If intermingled in forest areas, unimproved roads and nonforest strips must be more than 120 feet wide and more than 1 acre in area to qualify as nonforest land.)

a. Nonforest land without trees.-Nonforest land with no live trees present.

b. Nonforest land with trees. - Nonforest land with one or more trees at least 5 inches d.b.h. per acre.

Nonstocked land. - Commercial forest land less than 16.7 percent stocked with growing-stock trees.

Physiographic class.-A measure of soil and water conditions that affect tree growth on a site. Physiographic classes used in Resources Evaluation inventories are:

Xeric sites.-Very dry soils where excessive drainage seriously limits both growth and species occurrence. Example: sandy jack pine plains.

Xeromesic sites.-Moderately dry soils where excessive drainage limits growth and species occurrence to some extent. Example: dry oak ridge.

Mesic sites.-Deep, well-drained soils. Growth and species occurrence are limited only by climate.

Hydromesic sites. - Moderately wet soils where insufficient drainage or infrequent flooding limits growth and species occurrence to some extent. Example: better drained bottomland hardwood sites.

Hydric sites.-Very wet sites where excess water seriously limits both growth and species occurrence. Example: wet, frequently flooded river bottoms and spruce bogs.

Productive-reserved forest land.-Forest land sufficiently productive to qualify as commercial forest land but withdrawn from timber utilization through statute, administration regulation, designation, or exclusive use for Christmas tree production, as indicated by annual shearing.

Rotten trees.-Live trees of commercial species that do not contain at least one 12 -foot saw log or two saw logs 8 feet or longer, now or prospectively, and/or do not meet Regional specifications for freedom from defect primarily because of rot; that is, when more than 50 percent of the cull volume in a tree is rotten.

Rough trees.-(a) Live trees of commercial species that do not contain at least one merchantable 12 foot saw log or two saw $\operatorname{logs} 8$ feet or longer, now or prospectively, and/or do not meet Regional specifications for freedom from defect primarily because of roughness or poor form, and (b) all live trees of noncommercial species. 
Site class. - A classification of forest land in terms of inherent capacity to grow crops of industrial wood based on fully stocked natural stands.

Site index.-An expression of forest site quality based on the height of a free-growing dominant or codominant tree of a representative species in the forest type at age 50 .

Stand. - A growth of trees on a minimum of 1 acre of forest land that is stocked by forest trees of any size.

Stand-age class. - Age of the main stand. Main stand refers to trees of the dominant forest type and stand-size class.

Stocking. - The degree of occupancy of land by trees, measured by basal area and/or the number of trees in a stand by size or age and spacing, compared to the basal area and/or number of trees required to fully utilize the growth potential of the land; that is, the stocking standard.

A stocking percent of 100 indicates full utilization of the site and is equivalent to 80 square feet of basal area per acre in trees 5 inches d.b.h. and larger. In a stand of trees less than 5 inches d.b.h., a stocking percent of 100 would indicate that the prèsent number of trees is sufficient to produce 80 square feet of basal area per acre when the trees reach 5inches d.b.h.
Stands are grouped into the following stocking classes:

Overstocked stands.--Stands in which stocking of trees is 134.0 percent or more.

Fully stocked stands. - Stands in which stocking of trees is from 101.0 to 133.9 percent.

Medium stocked stands. - Stands in which stocking of trees is from 61.0 to 100.9 percent.

Poorly stocked stands. - Stands in which stocking of trees is from 16.7 to 60.9 percent.

Nonstocked areas.-Commercial forest land on which stocking of trees is less than 16.7 percent.

Unproductive forest land.-Forest land incapable of producing 20 cubic feet per acre of annual growth or- of yielding crops of industrial wood under natural conditions because of adverse site conditions. (Note: Adverse conditions include shallow soils, dry climate, poor drainage, high elevation, steepness, and rockiness.

Water:-(a) Bureau of the Census.-Streams, sloughs, estuaries, and canals more than one-eighth of a statute mile wide; and lakes, reservoirs, and ponds more than 40 acres in area.

(b) Noncensus. - The same as the Bureau of the Census, except minimum width of streams, etc. is 120 feet and minimum size of lakes, etc. is 1 acre. 
Table 4.--Area of aspen type commercial forest land by site productivity class, potential achieved, and stand-age class, for growing-stock trees, based on STEMS, Minnesota, 1977든

\begin{tabular}{|c|c|c|c|c|c|c|c|c|c|c|}
\hline \multirow{2}{*}{$\begin{array}{l}\text { Site } \\
\text { productivity } \\
\left(\mathrm{ft}^{3} / \mathrm{ac} / \mathrm{yr}\right)\end{array}$} & \multirow{2}{*}{$\begin{array}{l}\text { Potential } \\
\text { achieved }\end{array}$} & \multirow{2}{*}{$\begin{array}{c}\text { Al1 } \\
\text { classes } \\
\end{array}$} & \multicolumn{8}{|c|}{ Stand-age class (years) } \\
\hline & & & $1-10$ & $11-20$ & $21-30$ & $31-40$ & $41-50$ & $51-60$ & $61-70$ & $71+$ \\
\hline & Percent & $-\cdots---$ & $\cdots \cdots$ & ---- & $-n-n$ & Thousa & nd acres & 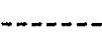 & - & ----- \\
\hline \multirow[t]{2}{*}{$101-120$} & $\begin{array}{r}0-25 \\
26-50 \\
51-75 \\
76+\end{array}$ & $\begin{array}{r}-- \\
5.4 \\
9.5 \\
6.8 \\
\end{array}$ & $\begin{array}{r}-- \\
1.4 \\
-- \\
\end{array}$ & $\begin{array}{l}-- \\
-- \\
-- \\
-\end{array}$ & $\begin{array}{r}-- \\
1.3 \\
--\overline{8} \\
\end{array}$ & $\begin{array}{r}-- \\
-- \\
-- \\
2.7\end{array}$ & $\begin{array}{r}-. \\
1.3 \\
2.8 \\
--\end{array}$ & $\begin{array}{r}-- \\
1.4 \\
3.9 \\
1.3 \\
\end{array}$ & $\begin{array}{r}-- \\
-- \\
1.4 \\
--\end{array}$ & $\begin{array}{r}-- \\
1.4 \\
-- \\
-- \\
\end{array}$ \\
\hline & Total & 21.7 & 1.4 & -- & 4.1 & 2.7 & 4.1 & 6.6 & 1.4 & 1.4 \\
\hline \multirow[t]{2}{*}{$81-100$} & $\begin{array}{r}0-25 \\
26-50 \\
51-75 \\
76+\end{array}$ & $\begin{array}{r}13.9 \\
87.3 \\
149.5 \\
110.5 \\
\end{array}$ & $\begin{array}{r}1.4 \\
1.2 \\
2.7 \\
14.9 \\
\end{array}$ & $\begin{array}{l}3.3 \\
2.7 \\
7.0 \\
4.4 \\
\end{array}$ & $\begin{array}{r}1.3 \\
16.2 \\
13.9 \\
20.2 \\
\end{array}$ & $\begin{array}{r}2.7 \\
17.3 \\
51.9 \\
24.8 \\
\end{array}$ & $\begin{array}{r}1.2 \\
25.8 \\
39.2 \\
24.4 \\
\end{array}$ & $\begin{array}{r}4.0 \\
11.8 \\
22.7 \\
9.6 \\
\end{array}$ & $\begin{array}{l}-- \\
9.6 \\
1.3 \\
2.7\end{array}$ & $\begin{array}{r}2 .- \\
2.7 \\
10.8 \\
9.5 \\
\end{array}$ \\
\hline & Total & 361.2 & 20.2 & 17.4 & 51.6 & 96.7 & 90.6 & 48.1 & 13.6 & 23.0 \\
\hline \multirow[t]{2}{*}{$61-80$} & $\begin{array}{r}0-25 \\
26-50 \\
51-75 \\
76+\end{array}$ & $\begin{array}{l}179.2 \\
867.0 \\
799.3 \\
512.7\end{array}$ & $\begin{array}{r}42.7 \\
34.5 \\
21.6 \\
165.8\end{array}$ & $\begin{array}{l}23.1 \\
49.6 \\
51.1 \\
54.1\end{array}$ & $\begin{array}{l}16.7 \\
85.1 \\
60.7 \\
32.7\end{array}$ & $\begin{array}{r}24.8 \\
193.3 \\
158.1 \\
75.0\end{array}$ & $\begin{array}{r}35.0 \\
240.2 \\
244.5 \\
87.8\end{array}$ & $\begin{array}{r}22.9 \\
183.7 \\
172.8 \\
66.3\end{array}$ & $\begin{array}{r}7.5 \\
43.7 \\
65.5 \\
17.4\end{array}$ & $\begin{array}{r}6.6 \\
37.0 \\
25.0 \\
13.8\end{array}$ \\
\hline & Total & $2,358.2$ & 264.6 & 177.9 & 195.1 & 451.2 & 607.5 & 445.6 & 134.0 & 82.4 \\
\hline \multirow[t]{2}{*}{$41-60$} & $\begin{array}{r}0-25 \\
26-50 \\
51-75 \\
76+\end{array}$ & $\begin{array}{l}408.5 \\
835.4 \\
599.3 \\
471.1 \\
\end{array}$ & $\begin{array}{r}110.0 \\
51.1 \\
67.5 \\
180.0 \\
\end{array}$ & $\begin{array}{l}64.4 \\
71.9 \\
83.6 \\
65.3 \\
\end{array}$ & $\begin{array}{r}50.8 \\
65.9 \\
38.2 \\
9.8 \\
\end{array}$ & $\begin{array}{l}51.0 \\
98.1 \\
70.3 \\
30.3\end{array}$ & $\begin{array}{r}45.4 \\
197.5 \\
131.7 \\
49.6\end{array}$ & $\begin{array}{r}56.8 \\
190.3 \\
124.2 \\
64.8 \\
\end{array}$ & $\begin{array}{l}16.5 \\
87.2 \\
53.7 \\
38.0\end{array}$ & $\begin{array}{l}13.7 \\
73.4 \\
30.2 \\
33.3\end{array}$ \\
\hline & Total & $2,314.4$ & 408.5 & 285.1 & 164.7 & 249.8 & 424.3 & 436.1 & 195.4 & 150.6 \\
\hline \multirow[t]{2}{*}{$21-40$} & $\begin{array}{r}0-25 \\
26-50 \\
51-75 \\
76+\end{array}$ & $\begin{array}{r}96.7 \\
115.4 \\
62.9 \\
46.7 \\
\end{array}$ & $\begin{array}{r}23.8 \\
9.3 \\
7.6 \\
21.1 \\
\end{array}$ & $\begin{array}{l}25.2 \\
12.4 \\
14.1 \\
14.1 \\
\end{array}$ & $\begin{array}{r}12.1 \\
9.0 \\
4.2 \\
\ldots\end{array}$ & $\begin{array}{r}14.4 \\
3.0 \\
5.7 \\
1.7\end{array}$ & $\begin{array}{r}7.2 \\
12.1 \\
14.5 \\
5.4\end{array}$ & $\begin{array}{r}9.9 \\
23.3 \\
8.4 \\
1.4 \\
\end{array}$ & $\begin{array}{r}1.3 \\
29.9 \\
2.8 \\
3.0\end{array}$ & $\begin{array}{r}2.8 \\
16.5 \\
5.5 \\
--\end{array}$ \\
\hline & Total & 321.7 & 61.9 & 65.8 & 25.3 & 24.8 & 39.1 & 43.0 & 37.0 & 24.8 \\
\hline \multirow[t]{2}{*}{ Total } & $\begin{array}{r}0-25 \\
26-50 \\
51-75 \\
76+\end{array}$ & $\begin{array}{r}698.4 \\
1,910.4 \\
1,620.6 \\
1,147.8 \\
\end{array}$ & $\begin{array}{r}177.9 \\
96.1 \\
100.9 \\
381.7 \\
\end{array}$ & $\begin{array}{l}115.9 \\
136.6 \\
155.9 \\
137.9\end{array}$ & $\begin{array}{r}80.9 \\
177.5 \\
117.0 \\
65.4 \\
\end{array}$ & $\begin{array}{r}92.9 \\
311.8 \\
286.0 \\
134.5\end{array}$ & $\begin{array}{r}88.7 \\
476.8 \\
432.8 \\
167.2\end{array}$ & $\begin{array}{r}93.6 \\
410.4 \\
332.0 \\
143.4\end{array}$ & $\begin{array}{r}25.3 \\
170.3 \\
124.7 \\
61.1\end{array}$ & $\begin{array}{r}23.1 \\
130.9 \\
71.5 \\
56.6\end{array}$ \\
\hline & Total & $5,377.2$ & 756.5 & 546.3 & 440.8 & 825.2 & $1,165.5$ & 979.4 & 381.4 & 282.1 \\
\hline
\end{tabular}

1/ Table may not add to totals due to rounding. Includes data from 1979 and 1980 for the Superior and Chippewa National Forests, respectively. 
Table 5.--Area of aspen type commercial forest land by site productivity class, potential achieved, and stand-age class, for all live trees, based on STEMS, Minnesota, 1977-

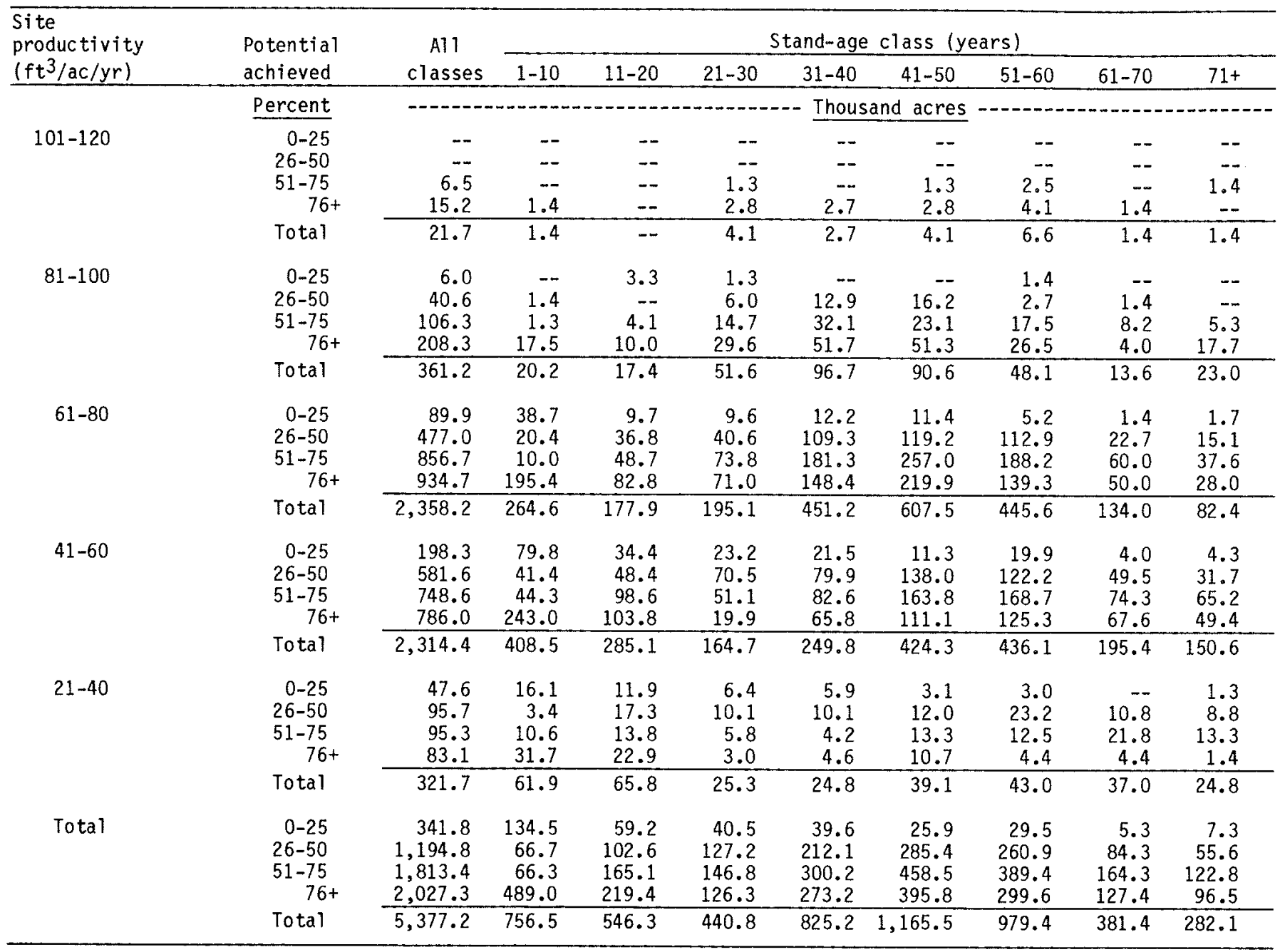

$1 /$ Table may not add to totals due to rounding. Includes data from 1979 and 1980 for the Superior and Chippewa National Forests, respectively. 
Table 6.--Area of aspen type comercial forest land by site productivity class, potential achieved, and physiographic class, for growing-stock trees, based on STEMS, Minnesota, 1977 I/

\begin{tabular}{|c|c|c|c|c|c|c|c|}
\hline \multirow{2}{*}{$\begin{array}{l}\text { Site } \\
\text { productivity } \\
\left(\mathrm{ft}^{3} / \mathrm{ac} / \mathrm{yr}\right)\end{array}$} & \multirow{2}{*}{$\begin{array}{l}\text { Potential } \\
\text { achieved }\end{array}$} & \multirow{2}{*}{$\begin{array}{c}\text { All } \\
\text { classes }\end{array}$} & \multicolumn{5}{|c|}{ Physiographic class } \\
\hline & & & Xeric & Xeromesic & Mesic & Hydromesic & Hydric \\
\hline \multirow[b]{2}{*}{$101-120$} & Percent & \multicolumn{6}{|c|}{ Thousand acres } \\
\hline & $\begin{array}{r}0-25 \\
26-50 \\
51-75 \\
76+\end{array}$ & $\begin{array}{r}-.- \\
5.4 \\
9.5 \\
6.8 \\
\end{array}$ & $\begin{array}{l}-- \\
-- \\
-- \\
\end{array}$ & $\begin{array}{l}- \\
- \\
-- \\
-- \\
-- \\
\end{array}$ & $\begin{array}{r}-- \\
4.1 \\
9.5 \\
6.8 \\
\end{array}$ & $\begin{array}{r}-- \\
1.3 \\
-.- \\
-- \\
\end{array}$ & $\begin{array}{l}-- \\
-- \\
-- \\
-- \\
\end{array}$ \\
\hline \multirow{3}{*}{$81-100$} & Total & 21.7 & -- & -- & 20.4 & 1.3 & -- \\
\hline & $\begin{array}{r}0-25 \\
26-50 \\
51-75 \\
76+\end{array}$ & $\begin{array}{r}13.9 \\
87.3 \\
149.5 \\
110.5 \\
\end{array}$ & $\begin{array}{l}-- \\
-- \\
-- \\
--\end{array}$ & $\begin{array}{l}-- \\
-- \\
1.4 \\
--\end{array}$ & $\begin{array}{r}8.7 \\
74.5 \\
141.2 \\
99.8\end{array}$ & $\begin{array}{r}5.2 \\
12.8 \\
6.9 \\
10.7\end{array}$ & $\begin{array}{l}-- \\
-- \\
-- \\
--\end{array}$ \\
\hline & Total & 361.2 & -- & 1.4 & 324.2 & 35.6 & -- \\
\hline \multirow[t]{2}{*}{$61-80$} & $\begin{array}{r}0-25 \\
26-50 \\
51-75 \\
76+\end{array}$ & $\begin{array}{l}179.2 \\
867.0 \\
799.3 \\
512.7 \\
\end{array}$ & $\begin{array}{l}-. . \\
2.1 \\
1.4 \\
1.5 \\
\end{array}$ & $\begin{array}{r}2.5 \\
11.9 \\
9.7 \\
1.4 \\
\end{array}$ & $\begin{array}{l}145.1 \\
744.7 \\
719.2 \\
467.8 \\
\end{array}$ & $\begin{array}{r}30.0 \\
102.6 \\
69.0 \\
42.0 \\
\end{array}$ & $\begin{array}{r}1.7 \\
5.7 \\
-- \\
-- \\
\end{array}$ \\
\hline & Total & $2,358.2$ & 5.0 & 25.5 & $2,076.8$ & 243.6 & 7.4 \\
\hline \multirow[t]{2}{*}{$41-60$} & $\begin{array}{r}0-25 \\
26-50 \\
51-75 \\
76+\end{array}$ & $\begin{array}{l}408.5 \\
835.4 \\
599.3 \\
471.1 \\
\end{array}$ & $\begin{array}{r}13.8 \\
1.4 \\
1.4 \\
-- \\
\end{array}$ & $\begin{array}{r}8.8 \\
10.6 \\
9.6 \\
12.9 \\
\end{array}$ & $\begin{array}{l}312.5 \\
697.0 \\
513.8 \\
413.7\end{array}$ & $\begin{array}{r}73.5 \\
122.0 \\
73.3 \\
42.8\end{array}$ & $\begin{array}{l}--- \\
4.5 \\
1.3 \\
1.7\end{array}$ \\
\hline & Total & $2,314.4$ & 16.6 & 41.8 & $1,937.0$ & 311.5 & 7.5 \\
\hline \multirow[t]{2}{*}{$21-40$} & $\begin{array}{r}0-25 \\
26-50 \\
51-75 \\
76+\end{array}$ & $\begin{array}{r}96.7 \\
115.4 \\
62.9 \\
46.7 \\
\end{array}$ & $\begin{array}{r}-- \\
1.4 \\
--- \\
--\end{array}$ & $\begin{array}{r}3.9 \\
4.2 \\
-- \\
3.0 \\
\end{array}$ & $\begin{array}{l}61.0 \\
88.0 \\
53.8 \\
31.2 \\
\end{array}$ & $\begin{array}{r}25.8 \\
21.8 \\
7.4 \\
12.5 \\
\end{array}$ & $\begin{array}{r}6.0 \\
-- \\
1.7 \\
-- \\
\end{array}$ \\
\hline & Total & 321.7 & 1.4 & 11.1 & 233.9 & 67.6 & 7.7 \\
\hline \multirow[t]{2}{*}{ Total } & $\begin{array}{r}0-25 \\
26-50 \\
51-75 \\
76+\end{array}$ & $\begin{array}{r}698.4 \\
1,910.4 \\
1,620.6 \\
1,147.8 \\
\end{array}$ & $\begin{array}{r}13.8 \\
4.9 \\
2.7 \\
1.5 \\
\end{array}$ & $\begin{array}{l}15.2 \\
26.7 \\
20.7 \\
17.3 \\
\end{array}$ & $\begin{array}{r}527.3 \\
1,608.2 \\
1,437.5 \\
1,019.3 \\
\end{array}$ & $\begin{array}{l}134.4 \\
260.5 \\
156.6 \\
108.0\end{array}$ & $\begin{array}{r}7.7 \\
10.2 \\
3.0 \\
1.7\end{array}$ \\
\hline & Total & $5,377.2$ & 22.9 & 79.8 & $4,592.3$ & 659.5 & 22.6 \\
\hline
\end{tabular}

$1 /$ Table may not add to totals due to rounding. Includes data from 1979 and 1980 for the Superior and Chippewa National Forests, respectively. 
Table 7.--Area of aspen type commercial forest land by site productivity class, potential achieved, and physiographic class, for all live trees, based on STEMS, Minnesota, 1977 1 /

\begin{tabular}{|c|c|c|c|c|c|c|c|}
\hline \multirow{2}{*}{$\begin{array}{l}\text { Site } \\
\text { productivity } \\
\left(\mathrm{ft}^{3} / \mathrm{ac} / \mathrm{yr}\right)\end{array}$} & \multirow{2}{*}{$\begin{array}{l}\text { Potential } \\
\text { achieved } \\
\end{array}$} & \multirow{2}{*}{$\begin{array}{c}\text { Al1 } \\
\text { classes } \\
\end{array}$} & \multicolumn{5}{|c|}{ Physiographic class } \\
\hline & & & Xeric & Xeromesic & Mesic & Hydromesic & Hydric \\
\hline \multirow{3}{*}{$101-120$} & Percent & $-\cdots-\cdots$ & $-\cdots$ & ..... Thou & and acres & $--n---n-1$ & 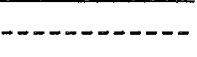 \\
\hline & $\begin{array}{r}0-25 \\
26-50 \\
51-75 \\
76+\end{array}$ & $\begin{array}{r}-- \\
-- \\
6.5 \\
15.2 \\
\end{array}$ & $\begin{array}{l}-- \\
-- \\
-- \\
-- \\
\end{array}$ & $\begin{array}{l}-- \\
-- \\
-- \\
-- \\
\end{array}$ & $\begin{array}{r}-- \\
-- \\
5.2 \\
15.2 \\
\end{array}$ & $\begin{array}{r}-- \\
-- \\
1.3 \\
-- \\
\end{array}$ & $\begin{array}{l}-- \\
-- \\
-- \\
-- \\
\end{array}$ \\
\hline & Total & 21.7 & -- & -- & 20.4 & 1.3 & -- \\
\hline \multirow[t]{2}{*}{$81-100$} & $\begin{array}{r}0-25 \\
26-50 \\
51-75 \\
76+\end{array}$ & $\begin{array}{r}6.0 \\
40.6 \\
106.3 \\
208.3 \\
\end{array}$ & $\begin{array}{l}-- \\
-- \\
-- \\
--\end{array}$ & $\begin{array}{r}-- \\
1.4 \\
-- \\
\end{array}$ & $\begin{array}{r}4.7 \\
33.6 \\
92.3 \\
193.6 \\
\end{array}$ & $\begin{array}{r}1.3 \\
7.0 \\
12.6 \\
14.7 \\
\end{array}$ & $\begin{array}{l}-- \\
-- \\
-- \\
--\end{array}$ \\
\hline & Total & 361.2 & -- & 1.4 & 324.2 & 35.6 & -- \\
\hline \multirow[t]{2}{*}{$61-80$} & $\begin{array}{r}0-25 \\
26-50 \\
51-75 \\
76+\end{array}$ & $\begin{array}{r}89.9 \\
477.0 \\
856.7 \\
934.7 \\
\end{array}$ & $\begin{array}{r}-- \\
-- \\
3.5 \\
1.5 \\
\end{array}$ & $\begin{array}{r}1.1 \\
10.3 \\
7.1 \\
7.0 \\
\end{array}$ & $\begin{array}{r}73.1 \\
392.2 \\
752.4 \\
859.1 \\
\end{array}$ & $\begin{array}{l}15.7 \\
68.7 \\
92.0 \\
67.2 \\
\end{array}$ & $\begin{array}{r}-- \\
5.8 \\
1.6 \\
-- \\
\end{array}$ \\
\hline & Total & $2,358.2$ & 5.0 & 25.5 & $2,076.8$ & 243.6 & 7.4 \\
\hline \multirow[t]{2}{*}{$41-60$} & $\begin{array}{r}0-25 \\
26-50 \\
51-75 \\
76+\end{array}$ & $\begin{array}{r}198.3 \\
581.6 \\
748.6 \\
786.0 \\
\end{array}$ & $\begin{array}{r}-- \\
15.2 \\
-\overline{-} \\
1.4 \\
\end{array}$ & $\begin{array}{r}5.8 \\
13.2 \\
7.2 \\
15.7 \\
\end{array}$ & $\begin{array}{l}160.5 \\
463.3 \\
628.5 \\
684.7 \\
\end{array}$ & $\begin{array}{r}32.0 \\
88.4 \\
108.7 \\
82.5 \\
\end{array}$ & $\begin{array}{l}-.- \\
1.6 \\
4.2 \\
1.7 \\
\end{array}$ \\
\hline & Total & $2,314.4$ & 16.6 & 41.8 & $1,937.0$ & 311.5 & 7.5 \\
\hline \multirow[t]{2}{*}{$21-40$} & $\begin{array}{r}0-25 \\
26-50 \\
51-75 \\
76+\end{array}$ & $\begin{array}{l}47.6 \\
95.7 \\
95.3 \\
83.1 \\
\end{array}$ & $\begin{array}{r}-- \\
1.4 \\
-- \\
-- \\
\end{array}$ & $\begin{array}{r}2.6 \\
4.2 \\
-- \\
4.3 \\
\end{array}$ & $\begin{array}{l}25.1 \\
72.9 \\
77.3 \\
58.6 \\
\end{array}$ & $\begin{array}{l}17.0 \\
15.5 \\
15.0 \\
20.1 \\
\end{array}$ & $\begin{array}{r}3.0 \\
1.7 \\
3.0 \\
-- \\
\end{array}$ \\
\hline & Total & 321.7 & 1.4 & 11.1 & 233.9 & 67.6 & 7.7 \\
\hline \multirow[t]{2}{*}{ Total } & $\begin{array}{r}0-25 \\
26-50 \\
51-75 \\
76+\end{array}$ & $\begin{array}{r}341.8 \\
1,194.8 \\
1,813.4 \\
2,027.3 \\
\end{array}$ & $\begin{array}{r}-- \\
16.6 \\
3.5 \\
2.9 \\
\end{array}$ & $\begin{array}{r}9.5 \\
27.7 \\
15.7 \\
27.0 \\
\end{array}$ & $\begin{array}{r}263.4 \\
961.9 \\
1,555.8 \\
1,811.2 \\
\end{array}$ & $\begin{array}{r}65.9 \\
179.5 \\
229.6 \\
184.5 \\
\end{array}$ & $\begin{array}{l}3.0 \\
9.1 \\
8.8 \\
1.7 \\
\end{array}$ \\
\hline & Total & $5,377.2$ & 22.9 & 79.8 & $4,592.3$ & 659.5 & 22.6 \\
\hline
\end{tabular}

$1 /$ Table may not add to totals due to rounding. Includes data from 1979 and 1980 for the Superior and Chippewa National Forests, respectively. 
Table 8.--Area of aspen type commercial forest land by site productivity class, potential achieved, and stand-age class, for growing-stock trees, based on STEMS, Aspen-Birch Unit, Minnesota, 1977 1 '

\begin{tabular}{|c|c|c|c|c|c|c|c|c|c|c|}
\hline \multirow{2}{*}{$\begin{array}{l}\text { Site } \\
\text { productivity } \\
\left(\mathrm{ft}^{3} / \mathrm{ac} / \mathrm{yr}\right)\end{array}$} & \multirow{2}{*}{$\begin{array}{l}\text { Potential } \\
\text { achieved }\end{array}$} & \multirow{2}{*}{$\begin{array}{c}\text { All } \\
\text { classes }\end{array}$} & \multicolumn{8}{|c|}{ Stand-age class (years) } \\
\hline & & & $1-10$ & $11-20$ & $21-30$ & $31-40$ & $41-50$ & $51-60$ & $61-70$ & $71+$ \\
\hline \multirow{3}{*}{$101-120$} & Percent & ------ & ---- & $-\cdots$ & $---\infty$ & Thousa & acres & ---- & $-\infty \cdots$ & ---- \\
\hline & $\begin{array}{r}0-25 \\
26-50 \\
51-75 \\
76+\end{array}$ & $\begin{array}{l}-- \\
1.4 \\
4.2 \\
2.7\end{array}$ & $\begin{array}{r}-- \\
1.4 \\
--\end{array}$ & $\begin{array}{l}-- \\
-- \\
-- \\
--\end{array}$ & $\begin{array}{l}-- \\
-- \\
-- \\
--\end{array}$ & $\begin{array}{l}-- \\
-- \\
2.7\end{array}$ & $\begin{array}{l}-- \\
2.8 \\
--\end{array}$ & $\begin{array}{l}-- \\
-- \\
--\end{array}$ & $\begin{array}{l}-- \\
-- \\
--\end{array}$ & $\begin{array}{l}-- \\
.1 \\
-\infty \\
--\end{array}$ \\
\hline & Total & 8.3 & 1.4 & -- & -- & 2.7 & 2.8 & -- & - & .1 \\
\hline \multirow[t]{2}{*}{$81-100$} & $\begin{array}{r}0-25 \\
26-50 \\
51-75 \\
76+\end{array}$ & $\begin{array}{r}2.8 \\
20.3 \\
43.1 \\
38.8\end{array}$ & $\begin{array}{r}1.4 \\
-- \\
-- \\
7.2\end{array}$ & $\begin{array}{r}-- \\
-- \\
4.2 \\
2.8\end{array}$ & $\begin{array}{l}-- \\
3.1 \\
4.3 \\
7.0\end{array}$ & $\begin{array}{r}-- \\
7.4 \\
13.7 \\
3.9\end{array}$ & $\begin{array}{r}-- \\
5.6 \\
12.4 \\
8.2\end{array}$ & $\begin{array}{l}1.4 \\
1.4 \\
5.9 \\
5.6\end{array}$ & $\begin{array}{r}-- \\
2.8 \\
-- \\
1.4\end{array}$ & $\begin{array}{r}-- \\
-- \\
2.6 \\
2.7\end{array}$ \\
\hline & Total & 105.0 & 8.6 & 7.0 & 14.4 & 25.0 & 26.2 & 14.3 & 4.2 & 5.3 \\
\hline \multirow[t]{2}{*}{$61-80$} & $\begin{array}{r}0-25 \\
26-50 \\
51-75 \\
76+\end{array}$ & $\begin{array}{r}52.9 \\
294.6 \\
256.0 \\
178.0\end{array}$ & $\begin{array}{r}10.0 \\
14.4 \\
6.1 \\
64.0 \\
\end{array}$ & $\begin{array}{l}4.6 \\
15.0 \\
14.1 \\
15.4 \\
\end{array}$ & $\begin{array}{r}9.0 \\
39.1 \\
19.4 \\
8.6 \\
\end{array}$ & $\begin{array}{r}8.5 \\
60.8 \\
47.5 \\
21.0 \\
\end{array}$ & $\begin{array}{l}12.3 \\
79.2 \\
71.8 \\
29.3\end{array}$ & $\begin{array}{r}7.0 \\
61.8 \\
68.7 \\
26.3 \\
\end{array}$ & $\begin{array}{r}1.5 \\
16.2 \\
21.9 \\
5.5\end{array}$ & $\begin{array}{l}-- \\
8.1 \\
6.5 \\
7.9\end{array}$ \\
\hline & Total & 781.5 & 94.5 & 49.1 & 76.1 & 137.8 & 192.6 & 163.8 & 45.1 & 22.5 \\
\hline \multirow[t]{2}{*}{$41-60$} & $\begin{array}{r}0-25 \\
26-50 \\
51-75 \\
76+\end{array}$ & $\begin{array}{l}160.8 \\
329.9 \\
222.8 \\
244.1\end{array}$ & $\begin{array}{l}35.9 \\
21.7 \\
28.8 \\
76.4\end{array}$ & $\begin{array}{l}21.1 \\
29.2 \\
30.2 \\
26.4\end{array}$ & $\begin{array}{r}27.7 \\
16.5 \\
16.7 \\
4.3\end{array}$ & $\begin{array}{l}18.4 \\
28.6 \\
17.4 \\
13.9\end{array}$ & $\begin{array}{l}19.8 \\
74.3 \\
31.5 \\
36.9\end{array}$ & $\begin{array}{l}28.2 \\
83.0 \\
57.3 \\
45.9\end{array}$ & $\begin{array}{l}1.4 \\
35.8 \\
21.9 \\
22.1\end{array}$ & $\begin{array}{r}8.3 \\
40.8 \\
19.0 \\
18.2\end{array}$ \\
\hline & Total & 957.6 & 162.8 & 106.9 & 65.2 & 78.3 & 162.5 & 214.4 & 81.2 & 86.3 \\
\hline \multirow[t]{2}{*}{$21-40$} & $\begin{array}{r}0-25 \\
26-50 \\
51-75 \\
76+\end{array}$ & $\begin{array}{l}22.8 \\
46.6 \\
18.1 \\
24.7\end{array}$ & $\begin{array}{r}4.0 \\
1.0 \\
-\overline{8.4}\end{array}$ & $\begin{array}{l}1.4 \\
5.6 \\
4.1 \\
9.5\end{array}$ & $\begin{array}{l}7.6 \\
2.8 \\
4.2 \\
--\end{array}$ & $\begin{array}{r}4.2 \\
-- \\
3.1 \\
--\end{array}$ & $\begin{array}{l}-.- \\
5.4 \\
2.6 \\
5.4\end{array}$ & $\begin{array}{r}4.1 \\
10.0 \\
4.1 \\
-.\end{array}$ & $\begin{array}{r}-\overline{-} \\
16.3 \\
1.4\end{array}$ & $\begin{array}{r}1.5 \\
5.5 \\
-- \\
--\end{array}$ \\
\hline & Total & 112.2 & 13.4 & 20.6 & 14.6 & 7.3 & 13.4 & 18.2 & 17.7 & 7.0 \\
\hline \multirow[t]{2}{*}{ Total } & $\begin{array}{r}0-25 \\
26-50 \\
51-75 \\
76+\end{array}$ & $\begin{array}{l}239.3 \\
692.8 \\
544.2 \\
488.3\end{array}$ & $\begin{array}{r}51.3 \\
37.1 \\
36.3 \\
156.0\end{array}$ & $\begin{array}{l}27.1 \\
49.8 \\
52.6 \\
54.1\end{array}$ & $\begin{array}{l}44.3 \\
61.5 \\
44.6 \\
19.9\end{array}$ & $\begin{array}{l}31.1 \\
96.8 \\
81.7 \\
41.5\end{array}$ & $\begin{array}{r}32.1 \\
164.5 \\
121.1 \\
79.8\end{array}$ & $\begin{array}{r}40.7 \\
156.2 \\
136.0 \\
77.8\end{array}$ & $\begin{array}{r}2.9 \\
71.1 \\
43.8 \\
30.4\end{array}$ & $\begin{array}{r}9.8 \\
55.8 \\
28.1 \\
28.8\end{array}$ \\
\hline & Total & $1,964.6$ & 280.7 & 183.6 & 170.3 & 251.1 & 397.5 & 410.7 & 148.2 & 122.5 \\
\hline
\end{tabular}

1/Table may not add to totals due to rounding. Includes data from 1979 for the Superior National Forest. 


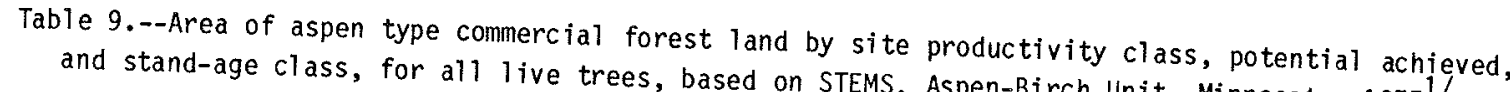
and stand-age class, for all live trees, based on STEMS, Aspen-Birch Unit, Minnesota, 1977 1 /

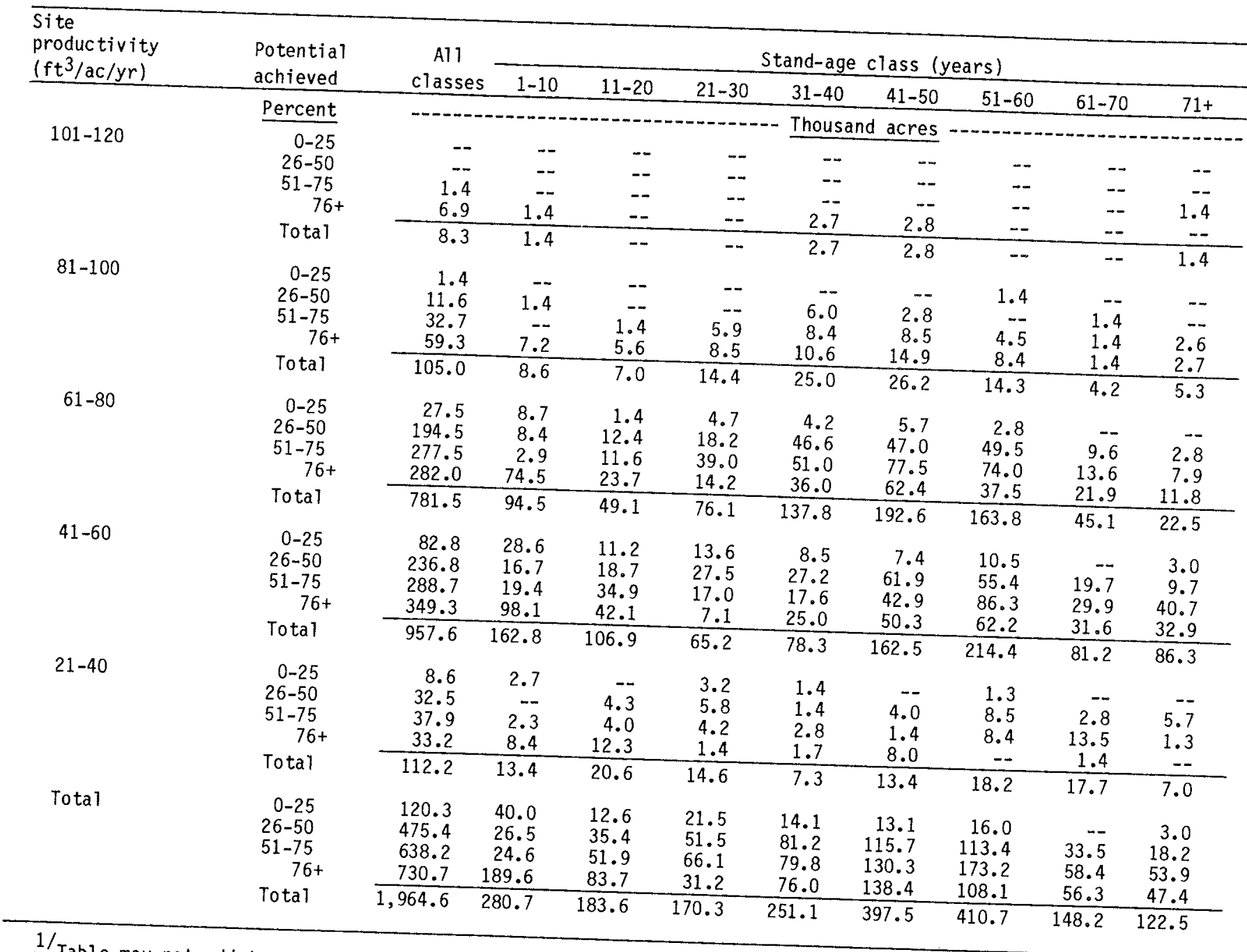

1 Table may not add to totals due to rounding. Includes data from 1979 for the Superior National Forest. 
Table 10.--Area of aspen type commercial forest land by site productivity class, potential achieved, and physiographic class, for growing-stock trees, based on STEMS, Aspen-Birch Unit, Minnesota, 1977 1 \}

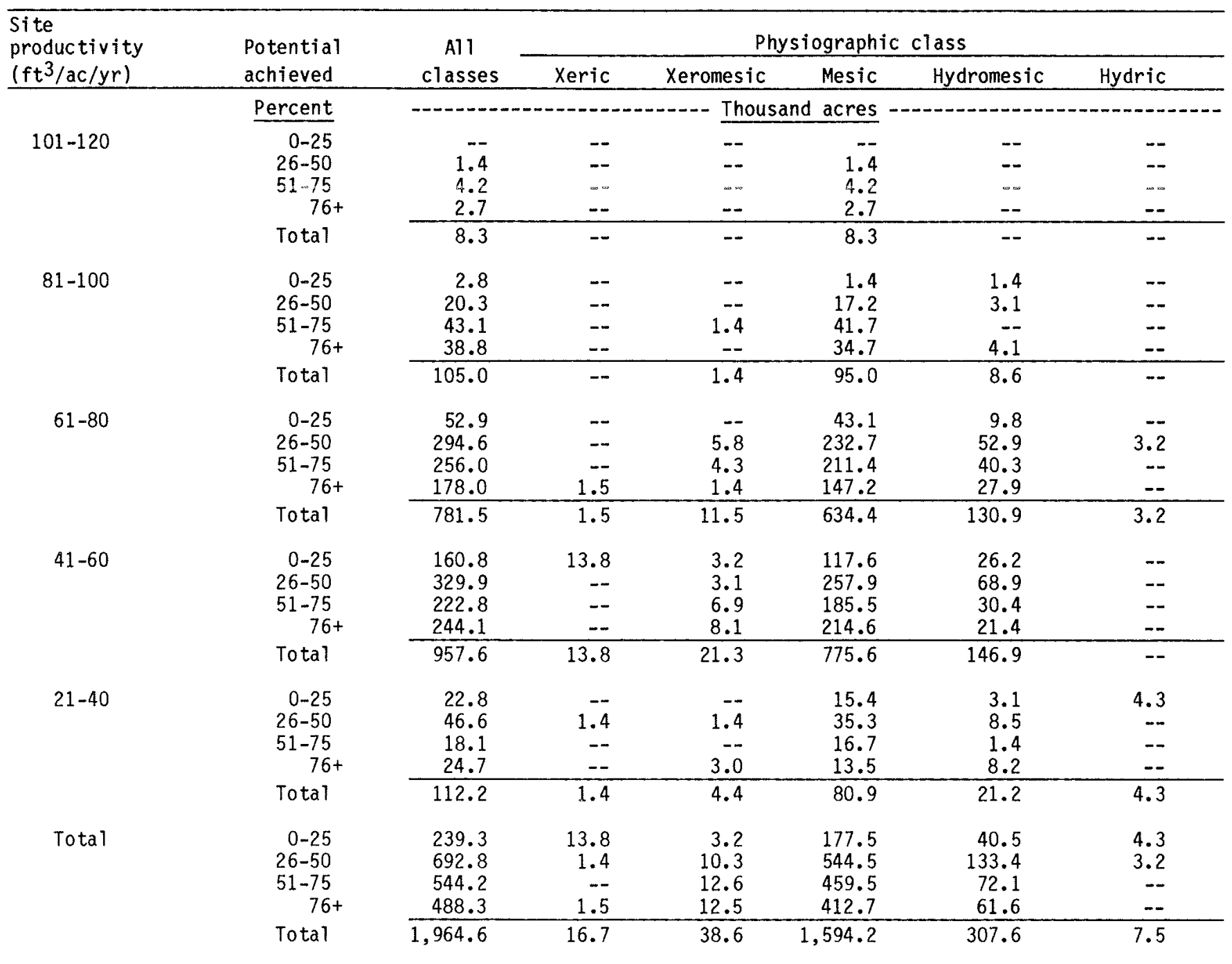

1/ Table may not add to totals due to rounding. Includes data from 1979 for the Superior National Forest. 
Table 11.--Area of aspen type commercial forest land by site productivity class, potential achieved, and physiographic class, for al1 live trees, based on STEMS, Aspen-Birch Unit, Minnesota, 1977-1/

\begin{tabular}{|c|c|c|c|c|c|c|c|}
\hline \multirow{2}{*}{$\begin{array}{l}\text { Site } \\
\text { productivity } \\
\left(\mathrm{ft}^{3} / \mathrm{ac} / \mathrm{yr}\right)\end{array}$} & \multirow{2}{*}{$\begin{array}{l}\text { Potential } \\
\text { achieved }\end{array}$} & \multirow{2}{*}{$\begin{array}{c}\text { A11 } \\
\text { classes } \\
\end{array}$} & \multicolumn{5}{|c|}{ Physiographic class } \\
\hline & & & Xeric & Xeromesic & Mesic & Hydromesic & Hydric \\
\hline \multirow{3}{*}{$101-120$} & Percent & 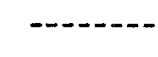 & ----- & Thous & d acres & $---1-n-1$ & ----- \\
\hline & $\begin{array}{r}0-25 \\
26-50 \\
51-75 \\
76+\end{array}$ & $\begin{array}{r}-- \\
1 .- \\
6.9 \\
\end{array}$ & $\begin{array}{l}-- \\
-- \\
-- \\
--\end{array}$ & $\begin{array}{l}-- \\
-- \\
-- \\
-- \\
\end{array}$ & $\begin{array}{r}-- \\
-- \\
1.4 \\
6.9 \\
\end{array}$ & $\begin{array}{l}-- \\
-- \\
-- \\
-- \\
\end{array}$ & $\begin{array}{l}-- \\
-- \\
-- \\
-\end{array}$ \\
\hline & Total & 8.3 & - & -- & 8.3 & - & - \\
\hline \multirow[t]{2}{*}{$31-100$} & $\begin{array}{r}0-25 \\
26-50 \\
51-75 \\
76+\end{array}$ & $\begin{array}{r}1.4 \\
11.6 \\
32.7 \\
59.3 \\
\end{array}$ & $\begin{array}{l}-- \\
-- \\
-- \\
--\end{array}$ & $\begin{array}{r}-- \\
-\overline{-} \\
1.4 \\
-- \\
\end{array}$ & $\begin{array}{r}1.4 \\
7.1 \\
31.3 \\
55.2 \\
\end{array}$ & $\begin{array}{r}--\overline{5} \\
4 .-\overline{1} \\
4.1\end{array}$ & $\begin{array}{l}-- \\
-- \\
--\end{array}$ \\
\hline & Total & 105.0 & -- & 1.4 & 95.0 & 8.6 & $\cdots$ \\
\hline \multirow[t]{2}{*}{$61-80$} & $\begin{array}{r}0-25 \\
26-50 \\
51-75 \\
76+\end{array}$ & $\begin{array}{r}27.5 \\
194.5 \\
277.5 \\
282.0 \\
\end{array}$ & $\begin{array}{l}-- \\
-- \\
1.5\end{array}$ & $\begin{array}{r}-. \overline{-} \\
4.4 \\
2.8 \\
4.3 \\
\end{array}$ & $\begin{array}{r}23.3 \\
143.1 \\
226.9 \\
241.1 \\
\end{array}$ & $\begin{array}{r}4.2 \\
45.4 \\
46.2 \\
35.1 \\
\end{array}$ & $\begin{array}{r}-- \\
1.6 \\
1.6 \\
--\end{array}$ \\
\hline & Total & 781.5 & 1.5 & 11.5 & 634.4 & 130.9 & 3.2 \\
\hline \multirow[t]{2}{*}{$41-60$} & $\begin{array}{r}0-25 \\
26-50 \\
51-75 \\
76+\end{array}$ & $\begin{array}{r}82.8 \\
236.8 \\
288.7 \\
349.3 \\
\end{array}$ & $\begin{array}{r}-- \\
13.8 \\
-- \\
-- \\
\end{array}$ & $\begin{array}{r}1.6 \\
4.4 \\
4.4 \\
10.9 \\
\end{array}$ & $\begin{array}{r}70.7 \\
175.6 \\
227.6 \\
301.7 \\
\end{array}$ & $\begin{array}{l}10.5 \\
43.0 \\
56.7 \\
36.7 \\
\end{array}$ & $\begin{array}{l}-- \\
-- \\
-- \\
-\end{array}$ \\
\hline & Total & 957.6 & 13.8 & 21.3 & 775.6 & 146.9 & -- \\
\hline \multirow[t]{2}{*}{$21-40$} & $\begin{array}{r}0-25 \\
26-50 \\
51-75 \\
76+\end{array}$ & $\begin{array}{r}8.6 \\
32.5 \\
37.9 \\
33.2 \\
\end{array}$ & $\begin{array}{l}-- \\
1.4 \\
-- \\
--\end{array}$ & $\begin{array}{r}-\overline{4} \\
-\overline{-} \\
3.0\end{array}$ & $\begin{array}{r}4.0 \\
23.9 \\
31.0 \\
22.0 \\
\end{array}$ & $\begin{array}{l}1.6 \\
5.8 \\
5.6 \\
8.2 \\
\end{array}$ & $\begin{array}{r}3.0 \\
-. \\
1.3 \\
-. \\
\end{array}$ \\
\hline & Total & 112.2 & 1.4 & 4.4 & 80.9 & 21.2 & 4.3 \\
\hline \multirow[t]{2}{*}{ Total } & $\begin{array}{r}0-25 \\
26-50 \\
51-75 \\
76+\end{array}$ & $\begin{array}{r}120.3 \\
475.4 \\
638.2 \\
730.7 \\
\end{array}$ & $\begin{array}{r}15 . \overline{-} \\
-- \\
1.5 \\
\end{array}$ & $\begin{array}{r}1.6 \\
10.2 \\
8.6 \\
18.2 \\
\end{array}$ & $\begin{array}{r}99.4 \\
349.7 \\
518.2 \\
626.9 \\
\end{array}$ & $\begin{array}{r}16.3 \\
98.7 \\
108.5 \\
84.1 \\
\end{array}$ & $\begin{array}{r}3.0 \\
1.6 \\
2.9 \\
-- \\
\end{array}$ \\
\hline & Total & $1,964.6$ & 16.7 & 38.6 & $1,594.2$ & 307.6 & 7.5 \\
\hline
\end{tabular}

$1 /$ Table may not add to totals due to rounding. Includes data from 1979 for the Superior National Forest. 
Table 12.--Area of aspen type commercial forest land by site productivity class, potential achieved, and stand-age class, for growing-stock trees, based on STEMS, Northern Pine Unit, Minnesota, 1977-1

\begin{tabular}{|c|c|c|c|c|c|c|c|c|c|c|}
\hline \multirow{2}{*}{$\begin{array}{l}\text { Site } \\
\text { productivity } \\
\left(\mathrm{ft}^{3} / \mathrm{ac} / \mathrm{yr}\right)\end{array}$} & \multirow{2}{*}{$\begin{array}{l}\text { Potential } \\
\text { achieved }\end{array}$} & \multirow{2}{*}{$\begin{array}{c}\text { A11 } \\
\text { classes }\end{array}$} & \multicolumn{8}{|c|}{ Stand-age class (years) } \\
\hline & & & $1-10$ & $11-20$ & $21-30$ & $31-40$ & $41-50$ & $51-60$ & $61-70$ & $71+$ \\
\hline \multirow{3}{*}{$101-120$} & Percent & ----- & $-\ldots$ & $\ldots-\ldots$ & 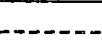 & - Thous & d acres & $-\ldots$ & $\ldots$ & $\ldots$ \\
\hline & $\begin{array}{r}0-25 \\
26-50 \\
51-75 \\
76+\end{array}$ & $\begin{array}{r}-.0 \\
5.0 \\
4.3 \\
\end{array}$ & $\begin{array}{l}-- \\
-- \\
-- \\
\end{array}$ & $\begin{array}{l}-- \\
-- \\
-- \\
\end{array}$ & $\begin{array}{r}-- \\
1.3 \\
-- \\
2.8 \\
\end{array}$ & $\begin{array}{l}-- \\
-- \\
-- \\
\end{array}$ & $\begin{array}{r}-- \\
1.3 \\
-- \\
-- \\
\end{array}$ & $\begin{array}{l}-- \\
1.4 \\
3.9 \\
1.3 \\
\end{array}$ & $\begin{array}{r}-- \\
-- \\
1.4 \\
--\end{array}$ & $\begin{array}{l}-- \\
-- \\
-- \\
-- \\
\end{array}$ \\
\hline & Total & 13.4 & - & -- & 4.1 & -- & 1.3 & 6.6 & 1.4 & -- \\
\hline \multirow[t]{2}{*}{$81-100$} & $\begin{array}{r}0-25 \\
26-50 \\
51-75 \\
76+\end{array}$ & $\begin{array}{r}9.8 \\
62.4 \\
103.6 \\
70.1 \\
\end{array}$ & $\begin{array}{l}-.- \\
1.2 \\
2.7 \\
7.7\end{array}$ & $\begin{array}{r}3.3 \\
2.7 \\
2.8 \\
-- \\
\end{array}$ & $\begin{array}{r}-.- \\
13.1 \\
9.6 \\
13.2 \\
\end{array}$ & $\begin{array}{r}2.7 \\
6.7 \\
35.4 \\
20.9 \\
\end{array}$ & $\begin{array}{r}1.2 \\
18.8 \\
26.8 \\
16.2\end{array}$ & $\begin{array}{r}2.6 \\
10.4 \\
16.8 \\
4.0\end{array}$ & $\begin{array}{l}-- \\
6.8 \\
1.3 \\
1.3\end{array}$ & $\begin{array}{l}-- \\
2.7 \\
8.2 \\
6.8\end{array}$ \\
\hline & Total & 245.9 & 11.6 & 8.8 & 35.9 & 65.7 & 63.0 & 33.8 & 9.4 & 17.7 \\
\hline \multirow[t]{2}{*}{$61-80$} & $\begin{array}{r}0-25 \\
26-50 \\
51-75 \\
76+\end{array}$ & $\begin{array}{r}107.8 \\
451.3 \\
468.1 \\
284.4 \\
\end{array}$ & $\begin{array}{r}31.1 \\
14.5 \\
9.7 \\
80.6 \\
\end{array}$ & $\begin{array}{l}12.8 \\
27.1 \\
30.4 \\
34.9 \\
\end{array}$ & $\begin{array}{r}7.2 \\
34.8 \\
30.4 \\
16.9 \\
\end{array}$ & $\begin{array}{l}15.0 \\
96.7 \\
82.6 \\
45.1 \\
\end{array}$ & $\begin{array}{r}15.7 \\
121.4 \\
151.8 \\
52.6 \\
\end{array}$ & $\begin{array}{r}14.8 \\
104.7 \\
101.1 \\
38.6\end{array}$ & $\begin{array}{r}4.6 \\
24.5 \\
43.6 \\
11.9\end{array}$ & $\begin{array}{r}6.6 \\
27.6 \\
18.5 \\
3.8\end{array}$ \\
\hline & Total & $\overline{1,311.6}$ & 135.9 & 105.2 & 89.3 & 239.4 & 341.5 & 259.2 & 84.6 & 56.5 \\
\hline \multirow[t]{2}{*}{$41-60$} & $\begin{array}{r}0-25 \\
26-50 \\
51-75 \\
76+\end{array}$ & $\begin{array}{l}145.8 \\
325.5 \\
273.5 \\
147.2 \\
\end{array}$ & $\begin{array}{l}46.1 \\
20.4 \\
17.6 \\
60.0 \\
\end{array}$ & $\begin{array}{l}31.2 \\
27.5 \\
42.4 \\
26.2 \\
\end{array}$ & $\begin{array}{r}14.7 \\
38.1 \\
15.5 \\
2.7 \\
\end{array}$ & $\begin{array}{r}10.6 \\
32.7 \\
36.8 \\
5.5 \\
\end{array}$ & $\begin{array}{r}13.9 \\
61.3 \\
71.6 \\
9.7 \\
\end{array}$ & $\begin{array}{l}15.8 \\
78.4 \\
51.0 \\
14.8 \\
\end{array}$ & $\begin{array}{r}9.4 \\
38.7 \\
29.0 \\
13.2 \\
\end{array}$ & $\begin{array}{r}4.1 \\
28.4 \\
9.6 \\
15.1 \\
\end{array}$ \\
\hline & Total & 892.0 & 144.1 & 127.3 & 71.0 & 85.6 & 156.5 & 160.0 & 90.3 & 57.2 \\
\hline \multirow[t]{2}{*}{$21-40$} & $\begin{array}{r}0-25 \\
26-50 \\
51-75 \\
76+\end{array}$ & $\begin{array}{l}44.3 \\
42.0 \\
25.0 \\
16.4 \\
\end{array}$ & $\begin{array}{r}13.4 \\
6.6 \\
6.0 \\
8.7 \\
\end{array}$ & $\begin{array}{r}16.1 \\
5.5 \\
2.8 \\
3.0 \\
\end{array}$ & $\begin{array}{r}1.6 \\
4.8 \\
-- \\
-- \\
\end{array}$ & $\begin{array}{l}6.0 \\
1.4 \\
1.2 \\
1.7 \\
\end{array}$ & $\begin{array}{r}4.4 \\
1.4 \\
6.6 \\
-- \\
\end{array}$ & $\begin{array}{l}2.8 \\
5.6 \\
1.4 \\
1.4 \\
\end{array}$ & $\begin{array}{l}-- \\
9.5 \\
2.8 \\
1.6 \\
\end{array}$ & $\begin{array}{r}-- \\
7.2 \\
4.2 \\
-- \\
\end{array}$ \\
\hline & Total & 127.7 & 34.7 & 27.4 & 6.4 & 10.3 & 12.4 & 11.2 & 13.9 & 11.4 \\
\hline \multirow[t]{2}{*}{ Total } & $\begin{array}{r}0-25 \\
26-50 \\
51-75 \\
76+\end{array}$ & $\begin{array}{r}307.7 \\
885.2 \\
875.5 \\
522.2 \\
\end{array}$ & $\begin{array}{r}90.6 \\
42.7 \\
36.0 \\
157.0 \\
\end{array}$ & $\begin{array}{l}63.4 \\
62.8 \\
78.4 \\
64.1 \\
\end{array}$ & $\begin{array}{l}23.5 \\
92.1 \\
55.5 \\
35.6\end{array}$ & $\begin{array}{r}34.3 \\
137.5 \\
156.0 \\
73.2 \\
\end{array}$ & $\begin{array}{r}35.2 \\
204.2 \\
256.8 \\
78.5\end{array}$ & $\begin{array}{r}36.0 \\
200.5 \\
174.2 \\
60.1\end{array}$ & $\begin{array}{l}14.0 \\
79.5 \\
78.1 \\
28.0\end{array}$ & $\begin{array}{l}10.7 \\
65.9 \\
40.5 \\
25.7\end{array}$ \\
\hline & Total & $2,590.6$ & 326.3 & 268.7 & 206.7 & 401.0 & 574.7 & 470.8 & 199.6 & 142.8 \\
\hline
\end{tabular}

1/Table may not add to totals due to rounding. Includes data from 1980 for the Chippewa National Forest. 
Table 13.--Area of aspen type commercial forest land by site productivity class, potential achieved, and stand-age class, for a11 1ive trees, based on STEMS, Northern Pine Unit, Minnesota, 1977 1/

\begin{tabular}{|c|c|c|c|c|c|c|c|c|c|c|}
\hline \multirow{2}{*}{$\begin{array}{l}\text { Site } \\
\text { productivity } \\
\left(\mathrm{ft}^{3} / \mathrm{ac} / \mathrm{yr}\right)\end{array}$} & \multirow{2}{*}{$\begin{array}{l}\text { Potential } \\
\text { achieved }\end{array}$} & \multirow{2}{*}{$\begin{array}{c}\text { All } \\
\text { classes } \\
\end{array}$} & \multicolumn{8}{|c|}{ Stand-age class (years) } \\
\hline & & & $1-10$ & $11-20$ & $21-30$ & $31-40$ & $41-50$ & $51-60$ & $61-70$ & $71+$ \\
\hline \multirow{3}{*}{$101-120$} & Percent & ----- & $-\cdots$ & --..- & $-\ldots-.-$ & Thousa & acres & $-\ldots$ & $\ldots \ldots$ & $-\ldots$ \\
\hline & $\begin{array}{c}0-25 \\
26-50 \\
51-75 \\
76+\end{array}$ & $\begin{array}{r}-- \\
-- \\
8.1 \\
8.3 \\
\end{array}$ & $\begin{array}{l}-- \\
- \\
-- \\
\end{array}$ & $\begin{array}{l}-- \\
-- \\
-- \\
-\end{array}$ & $\begin{array}{r}-- \\
-- \\
1.3 \\
2.8 \\
\end{array}$ & $\begin{array}{l}-- \\
-- \\
-- \\
-- \\
\end{array}$ & $\begin{array}{r}-- \\
1 . \overline{3} \\
--\end{array}$ & $\begin{array}{r}-- \\
-- \\
2.5 \\
4.1 \\
\end{array}$ & $\begin{array}{l}-- \\
-- \\
1.4 \\
\end{array}$ & $\begin{array}{l}-- \\
-- \\
-- \\
\end{array}$ \\
\hline & Total & 13.4 & -- & - & 4.1 & -- & 1.3 & 6.6 & 1.4 & -- \\
\hline \multirow[t]{2}{*}{$81-100$} & $\begin{array}{r}0-25 \\
26-50 \\
51-75 \\
76+\end{array}$ & $\begin{array}{r}3.3 \\
26.0 \\
69.2 \\
147.4 \\
\end{array}$ & $\begin{array}{r}-. . \\
1.3 \\
10.3 \\
\end{array}$ & $\begin{array}{r}3.3 \\
-- \\
2.7 \\
2.8 \\
\end{array}$ & $\begin{array}{r}-- \\
5.0 \\
8.8 \\
21.1 \\
\end{array}$ & $\begin{array}{r}5 . \overline{3} \\
19.3 \\
41.1 \\
\end{array}$ & $\begin{array}{l}-- \\
12.0 \\
14.6 \\
36.4 \\
\end{array}$ & $\begin{array}{r}-.7 \\
13.7 \\
18.0 \\
\end{array}$ & $\begin{array}{r}-- \\
-- \\
6.8 \\
2.6 \\
\end{array}$ & $\begin{array}{r}-- \\
-- \\
2.7 \\
15.0 \\
\end{array}$ \\
\hline & Total & 245.9 & 11.6 & 8.8 & 35.9 & 65.7 & 63.0 & 33.8 & 9.4 & 17.7 \\
\hline \multirow[t]{2}{*}{$61-80$} & $\begin{array}{r}0-25 \\
26-50 \\
51-75 \\
76+\end{array}$ & $\begin{array}{r}53.0 \\
231.4 \\
473.1 \\
554.1 \\
\end{array}$ & $\begin{array}{r}28.4 \\
7.7 \\
5.5 \\
94.3 \\
\end{array}$ & $\begin{array}{r}5.1 \\
17.6 \\
29.3 \\
53.2 \\
\end{array}$ & $\begin{array}{r}4.4 \\
19.8 \\
26.5 \\
38.6 \\
\end{array}$ & $\begin{array}{r}6.7 \\
48.9 \\
93.0 \\
90.8 \\
\end{array}$ & $\begin{array}{r}4.3 \\
57.9 \\
143.1 \\
136.2 \\
\end{array}$ & $\begin{array}{r}2.4 \\
55.4 \\
102.6 \\
98.8 \\
\end{array}$ & $\begin{array}{l}--- \\
13.1 \\
43.4 \\
28.1 \\
\end{array}$ & $\begin{array}{r}1.7 \\
11.0 \\
29.7 \\
14.1 \\
\end{array}$ \\
\hline & Total & $\overline{1,311.6}$ & 135.9 & 105.2 & 89.3 & 239.4 & 341.5 & 259.2 & 84.6 & 56.5 \\
\hline \multirow[t]{2}{*}{$41-60$} & $\begin{array}{r}0-25 \\
26-50 \\
51-75 \\
76\end{array}$ & $\begin{array}{r}76.1 \\
216.9 \\
315.0 \\
284.0 \\
\end{array}$ & $\begin{array}{l}37.4 \\
16.8 \\
14.5 \\
75.4 \\
\end{array}$ & $\begin{array}{l}18.8 \\
21.2 \\
50.7 \\
36.6 \\
\end{array}$ & $\begin{array}{r}7.9 \\
26.0 \\
28.7 \\
8.4 \\
\end{array}$ & $\begin{array}{r}1.6 \\
25.4 \\
36.3 \\
22.3 \\
\end{array}$ & $\begin{array}{r}3.9 \\
37.5 \\
68.4 \\
46.7 \\
\end{array}$ & $\begin{array}{r}3.8 \\
48.3 \\
60.5 \\
47.4 \\
\end{array}$ & $\begin{array}{r}2.7 \\
19.7 \\
37.2 \\
30.7 \\
\end{array}$ & $\begin{array}{l}-- \\
22.0 \\
18.7 \\
16.5 \\
\end{array}$ \\
\hline & Total & 892.0 & 144.1 & 127.3 & 71.0 & 85.6 & 156.5 & 160.0 & 90.3 & 57.2 \\
\hline \multirow[t]{2}{*}{$21-40$} & $\begin{array}{r}0-25 \\
26-50 \\
51-75 \\
76+\end{array}$ & $\begin{array}{l}28.1 \\
30.3 \\
37.4 \\
31.9 \\
\end{array}$ & $\begin{array}{r}10.4 \\
1.7 \\
6.7 \\
15.9 \\
\end{array}$ & $\begin{array}{l}9.9 \\
7.3 \\
7.2 \\
3.0 \\
\end{array}$ & $\begin{array}{l}1.6 \\
1.6 \\
1.6 \\
1.6 \\
\end{array}$ & $\begin{array}{r}4.5 \\
2.9 \\
-- \\
2.9 \\
\end{array}$ & $\begin{array}{l}1.7 \\
2.7 \\
6.7 \\
1.3 \\
\end{array}$ & $\begin{array}{l}7.0 \\
1.4 \\
2.8 \\
\end{array}$ & $\begin{array}{l}-- \\
4.0 \\
6.9 \\
3.0 \\
\end{array}$ & $\begin{array}{l}3 .- \\
6.9 \\
1.4 \\
\end{array}$ \\
\hline & Total & 127.7 & 34.7 & 27.4 & 6.4 & 10.3 & 12.4 & 11.2 & 13.9 & 11.4 \\
\hline \multirow[t]{2}{*}{ Total } & $\begin{array}{r}0-25 \\
26-50 \\
51-75 \\
76+\end{array}$ & $\begin{array}{r}160.5 \\
504.6 \\
899.8 \\
1,025.7 \\
\end{array}$ & $\begin{array}{r}76.2 \\
26.2 \\
28.0 \\
195.9 \\
\end{array}$ & $\begin{array}{l}37.1 \\
46.1 \\
89.9 \\
95.6 \\
\end{array}$ & $\begin{array}{l}13.9 \\
53.4 \\
66.9 \\
72.5 \\
\end{array}$ & $\begin{array}{r}12.8 \\
82.5 \\
148.6 \\
157.1 \\
\end{array}$ & $\begin{array}{r}9.9 \\
110.1 \\
234.1 \\
220.6 \\
\end{array}$ & $\begin{array}{r}6.2 \\
113.4 \\
180.0 \\
171.2 \\
\end{array}$ & $\begin{array}{r}2.7 \\
36.8 \\
94.3 \\
65.8 \\
\end{array}$ & $\begin{array}{r}1.7 \\
36.1 \\
58.0 \\
47.0 \\
\end{array}$ \\
\hline & Total & $2,590.6$ & 326.3 & 268.7 & 206.7 & 401.0 & 574.7 & 470.8 & 199.6 & 142.8 \\
\hline
\end{tabular}

$1 /$ Table may not add to totals due to rounding. Includes data from 1980 for the Chippewa National Forest. 
Table 14.--Area of aspen type commercial forest land by site productivity class, potential achieved, and physiographic class, for growing-stock trees, based on STEMS, Northern Pine Unit, Minnesota, 1977 I/

\begin{tabular}{|c|c|c|c|c|c|c|c|}
\hline \multirow{2}{*}{$\begin{array}{l}\text { Site } \\
\text { productivity } \\
\left(\mathrm{ft}^{3} / \mathrm{ac} / \mathrm{yr}\right)\end{array}$} & \multirow{2}{*}{$\begin{array}{l}\text { Potential } \\
\text { achieved }\end{array}$} & \multirow{2}{*}{$\begin{array}{c}\text { Al1 } \\
\text { classes } \\
\end{array}$} & \multicolumn{5}{|c|}{ Physiographic class } \\
\hline & & & Xeric & Xeromesic & Mesic & Hydromesic & Hydric \\
\hline & Percent & $\ldots$ & $\ldots$ & ...- Thous & nd acres & 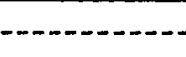 & 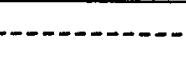 \\
\hline \multirow[t]{2}{*}{$101-120$} & $\begin{array}{r}0-25 \\
26-50 \\
51-75 \\
76+\end{array}$ & $\begin{array}{r}-- \\
4.0 \\
5.3 \\
4.1 \\
\end{array}$ & $\begin{array}{l}-- \\
-- \\
-- \\
--\end{array}$ & $\begin{array}{l}-- \\
-- \\
-- \\
-- \\
\end{array}$ & $\begin{array}{r}-.- \\
2.7 \\
5.3 \\
4.1 \\
\end{array}$ & $\begin{array}{r}-- \\
1.3 \\
-- \\
-- \\
\end{array}$ & $\begin{array}{l}-- \\
-- \\
-- \\
-- \\
\end{array}$ \\
\hline & Total & 13.4 & -- & -- & 12.1 & 1.3 & -- \\
\hline \multirow[t]{2}{*}{$81-100$} & $\begin{array}{r}0-25 \\
26-50 \\
51-75 \\
76+\end{array}$ & $\begin{array}{r}9.8 \\
62.4 \\
103.6 \\
70.1 \\
\end{array}$ & $\begin{array}{l}-- \\
-- \\
-- \\
--\end{array}$ & $\begin{array}{l}-- \\
-- \\
-- \\
--\end{array}$ & $\begin{array}{r}7.3 \\
54.3 \\
96.7 \\
63.5\end{array}$ & $\begin{array}{l}2.5 \\
8.1 \\
6.9 \\
6.6\end{array}$ & $\begin{array}{l}-- \\
-- \\
-- \\
--\end{array}$ \\
\hline & Total & 245.9 & -- & -- & 221.8 & 24.1 & -- \\
\hline \multirow[t]{2}{*}{$61-80$} & $\begin{array}{r}0-25 \\
26-50 \\
51-75 \\
76+\end{array}$ & $\begin{array}{r}107.8 \\
451.3 \\
468.1 \\
284.4 \\
\end{array}$ & $\begin{array}{l}-- \\
2.1 \\
-- \\
--\end{array}$ & $\begin{array}{r}2.5 \\
6.1 \\
5.4 \\
-- \\
\end{array}$ & $\begin{array}{r}89.1 \\
397.9 \\
438.0 \\
273.5 \\
\end{array}$ & $\begin{array}{l}14.5 \\
42.7 \\
24.7 \\
10.9\end{array}$ & $\begin{array}{r}1.7 \\
2.5 \\
-. \\
-.\end{array}$ \\
\hline & Total & $1,311.6$ & 2.1 & 14.0 & $1,198.5$ & 92.8 & 4.2 \\
\hline \multirow[t]{2}{*}{$41-60$} & $\begin{array}{r}0-25 \\
26-50 \\
51-75 \\
76+\end{array}$ & $\begin{array}{l}145.8 \\
325.5 \\
273.5 \\
147.2 \\
\end{array}$ & $\begin{array}{r}-. \\
1.4 \\
-- \\
--\end{array}$ & $\begin{array}{l}5.6 \\
5.9 \\
2.7 \\
3.1 \\
\end{array}$ & $\begin{array}{l}108.1 \\
277.6 \\
240.9 \\
127.8 \\
\end{array}$ & $\begin{array}{l}32.1 \\
37.7 \\
28.6 \\
14.6 \\
\end{array}$ & $\begin{array}{l}-- \\
2.9 \\
1.3 \\
1.7\end{array}$ \\
\hline & Total & 892.0 & 1.4 & 17.3 & 754.4 & 113.0 & 5.9 \\
\hline \multirow[t]{2}{*}{$21-40$} & $\begin{array}{r}0-25 \\
26-50 \\
51-75 \\
76+\end{array}$ & $\begin{array}{l}44.3 \\
42.0 \\
25.0 \\
16.4 \\
\end{array}$ & $\begin{array}{l}-- \\
-- \\
-- \\
--\end{array}$ & $\begin{array}{r}3.9 \\
2.8 \\
-- \\
-- \\
\end{array}$ & $\begin{array}{l}23.7 \\
28.2 \\
18.9 \\
13.4 \\
\end{array}$ & $\begin{array}{r}15.0 \\
11.0 \\
4.4 \\
3.0\end{array}$ & $\begin{array}{r}1.7 \\
-- \\
1.7 \\
--\end{array}$ \\
\hline & Total & 127.7 & $-\infty$ & 6.7 & 84.2 & 33.4 & 3.4 \\
\hline \multirow[t]{2}{*}{ Total } & $\begin{array}{r}0-25 \\
26-50 \\
51-75 \\
76+\end{array}$ & $\begin{array}{l}307.7 \\
885.2 \\
875.5 \\
522.2 \\
\end{array}$ & $\begin{array}{r}-- \\
3.5 \\
-- \\
--\end{array}$ & $\begin{array}{r}12.0 \\
14.8 \\
8.1 \\
3.1\end{array}$ & $\begin{array}{l}228.2 \\
760.7 \\
799.8 \\
482.3\end{array}$ & $\begin{array}{r}64.1 \\
100.8 \\
64.6 \\
35.1\end{array}$ & $\begin{array}{l}3.4 \\
5.4 \\
3.0 \\
1.7\end{array}$ \\
\hline & Total & $2,590.6$ & 3.5 & 38.0 & $2,271.0$ & 264.6 & 13.5 \\
\hline
\end{tabular}

1/ Table may not add to totals due to rounding. Includes data from 1980 for the Chippewa National Forest. 
Table 15.--Area of aspen type commercial forest land by site productivity class, potential achieved, and physiographic class, for all live trees, based on STEMS, Northern Pine Unit, Minnesota, 1977 $1 /$

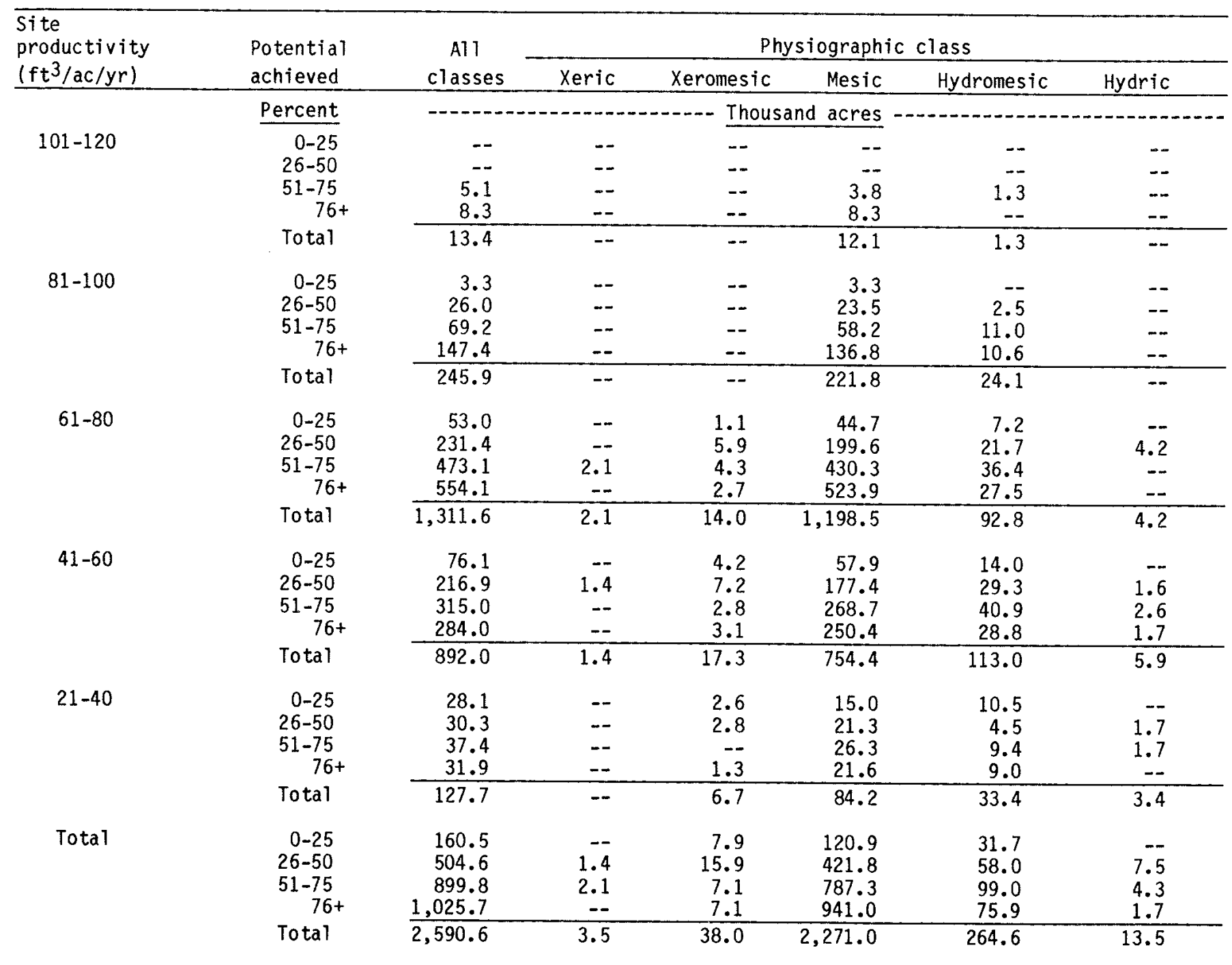

$1 /$ Table may not add to totals due to rounding. Includes data from 1980 for the Chippewa National Forest. 
Table 16.--Area of aspen type commercial forest land by site productivity class, potential achieved, and stand-age class, for growing-stock trees, based on STEMS, Central Hardwood Unit, Minnesota, 1977 1 -

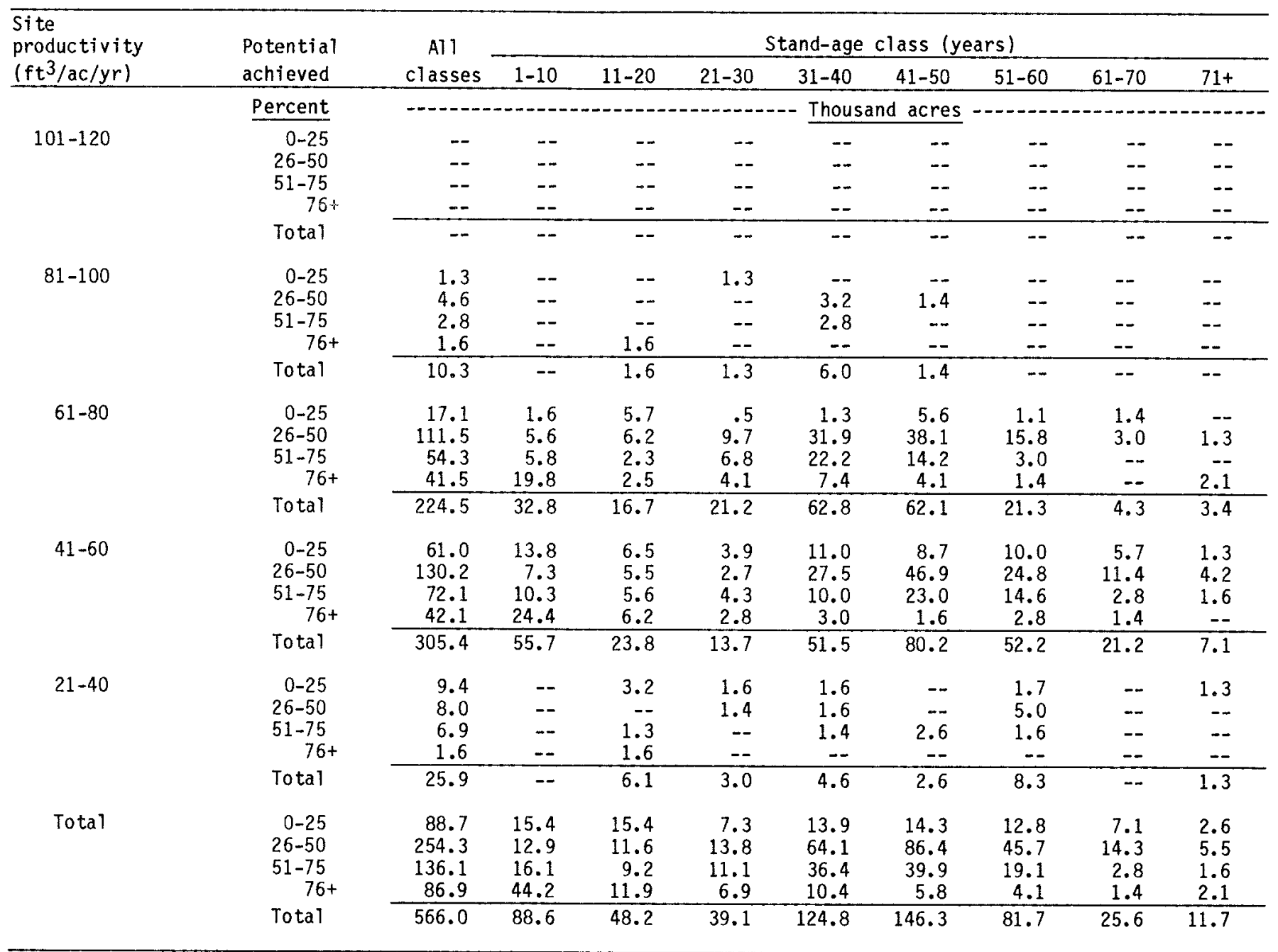

I/ Table may not add to totals due to rounding. 
Table 17.--Area of aspen type commercial forest land by site productivity class, potential achieved, and stand-age class, for all live trees, based on STEMS, Central Hardwood Unit, Minnesota, 1977-1/

\begin{tabular}{|c|c|c|c|c|c|c|c|c|c|c|}
\hline \multirow{2}{*}{$\begin{array}{l}\text { Site } \\
\text { productivity } \\
\left(\mathrm{ft}^{3} / \mathrm{ac} / \mathrm{yr}\right)\end{array}$} & \multirow{2}{*}{$\begin{array}{l}\text { Potential } \\
\text { achieved }\end{array}$} & \multirow{2}{*}{$\begin{array}{c}\text { All } \\
\text { classes }\end{array}$} & \multicolumn{8}{|c|}{ Stand-age class (years) } \\
\hline & & & $1-10$ & $11-20$ & $21-30$ & $31-40$ & $41-50$ & $51-60$ & $61-70$ & $71+$ \\
\hline \multirow{3}{*}{$101-120$} & Percent & 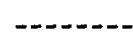 & ---- & 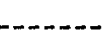 & 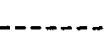 & Thousa & acres & $\ldots$ & $-\cdots$ & $-\ldots$ \\
\hline & $\begin{array}{r}0-25 \\
26-50 \\
51-75 \\
76+\end{array}$ & $\begin{array}{l}-- \\
-- \\
-- \\
--\end{array}$ & $\begin{array}{l}-- \\
-- \\
-- \\
-- \\
\end{array}$ & $\begin{array}{l}-- \\
-- \\
-- \\
-- \\
\end{array}$ & $\begin{array}{l}-- \\
-- \\
-- \\
--\end{array}$ & $\begin{array}{l}-- \\
-- \\
-- \\
\end{array}$ & $\begin{array}{l}-- \\
-- \\
-- \\
-\end{array}$ & $\begin{array}{l}-- \\
-- \\
-- \\
-\end{array}$ & $\begin{array}{l}-- \\
-- \\
-- \\
\end{array}$ & $\begin{array}{l}-- \\
-- \\
-- \\
\end{array}$ \\
\hline & Total & - & -- & -- & -- & - & -- & -- & -- & -- \\
\hline \multirow[t]{2}{*}{$81-100$} & $\begin{array}{r}0-25 \\
26-50 \\
51-75 \\
76+\end{array}$ & $\begin{array}{l}1.3 \\
3.0 \\
4.4 \\
1.6 \\
\end{array}$ & $\begin{array}{l}-- \\
-- \\
-- \\
--\end{array}$ & $\begin{array}{l}-- \\
-- \\
-- \\
1.6\end{array}$ & $\begin{array}{c}1.3 \\
-- \\
-- \\
-- \\
\end{array}$ & $\begin{array}{r}-- \\
1.6 \\
4.4 \\
-- \\
\end{array}$ & $\begin{array}{r}-- \\
1.4 \\
-- \\
--\end{array}$ & $\begin{array}{l}-- \\
-- \\
-- \\
--\end{array}$ & $\begin{array}{l}-- \\
-- \\
-- \\
--\end{array}$ & $\begin{array}{l}-- \\
-- \\
-- \\
--\end{array}$ \\
\hline & Total & 10.3 & -- & 1.6 & 1.3 & 6.0 & 1.4 & - & -- & -- \\
\hline \multirow[t]{2}{*}{$61-80$} & $\begin{array}{r}0-25 \\
26-50 \\
51-75 \\
76+\end{array}$ & $\begin{array}{r}9.4 \\
48.4 \\
93.8 \\
72.9 \\
\end{array}$ & $\begin{array}{r}1.6 \\
4.3 \\
1.6 \\
25.3 \\
\end{array}$ & $\begin{array}{l}3.2 \\
5.5 \\
4.7 \\
3.2 \\
\end{array}$ & $\begin{array}{l}.5 \\
2.6 \\
8.3 \\
9.7 \\
\end{array}$ & $\begin{array}{l}1.3 \\
12.4 \\
32.3 \\
16.8 \\
\end{array}$ & $\begin{array}{r}1.4 \\
14.3 \\
33.5 \\
12.8 \\
\end{array}$ & $\begin{array}{r}-- \\
8.0 \\
10.3 \\
3.0 \\
\end{array}$ & $\begin{array}{r}1.4 \\
-- \\
3.0 \\
-- \\
\end{array}$ & $\begin{array}{r}-- \\
1.3 \\
-\overline{-} \\
2.1\end{array}$ \\
\hline & Total & 224.5 & 32.8 & 16.7 & 21.2 & 62.8 & 62.1 & 21.3 & 4.3 & 3.4 \\
\hline \multirow[t]{2}{*}{$41-60$} & $\begin{array}{r}0-25 \\
26-50 \\
51-75 \\
76+\end{array}$ & $\begin{array}{r}24.0 \\
90.6 \\
101.6 \\
89.2 \\
\end{array}$ & $\begin{array}{r}6.0 \\
4.5 \\
7.4 \\
37.9 \\
\end{array}$ & $\begin{array}{r}3.3 \\
5.9 \\
4.0 \\
10.6 \\
\end{array}$ & $\begin{array}{l}-- \\
6.6 \\
2.7 \\
4.4 \\
\end{array}$ & $\begin{array}{r}6.6 \\
21.6 \\
14.1 \\
9.3 \\
\end{array}$ & $\begin{array}{r}-- \\
26.3 \\
44.0 \\
9.9 \\
\end{array}$ & $\begin{array}{r}5.6 \\
15.7 \\
17.9 \\
13.0 \\
\end{array}$ & $\begin{array}{r}1.3 \\
10.1 \\
5.8 \\
4.0 \\
\end{array}$ & $\begin{array}{r}1.3 \\
-- \\
5.8 \\
-- \\
\end{array}$ \\
\hline & Total & 305.4 & 55.7 & 23.8 & 13.7 & 51.5 & 80.2 & 52.2 & 21.2 & 7.1 \\
\hline \multirow[t]{2}{*}{$21-40$} & $\begin{array}{r}0-25 \\
26-50 \\
51-75 \\
76+\end{array}$ & $\begin{array}{r}6.5 \\
10.8 \\
3.9 \\
4.6 \\
\end{array}$ & $\begin{array}{l}-- \\
-- \\
-- \\
-- \\
\end{array}$ & $\begin{array}{l}2.0 \\
1.3 \\
1.3 \\
1.6 \\
\end{array}$ & $\begin{array}{r}1.6 \\
1.4 \\
-. \\
-. \\
\end{array}$ & $\begin{array}{r}-- \\
3.2 \\
1.4 \\
-- \\
\end{array}$ & $\begin{array}{r}-- \\
-- \\
1.3 \\
1.4 \\
\end{array}$ & $\begin{array}{r}1.7 \\
5.0 \\
-- \\
1.6 \\
\end{array}$ & $\begin{array}{l}-- \\
-- \\
-- \\
--\end{array}$ & $\begin{array}{c}1.3 \\
-- \\
-- \\
-. \\
\end{array}$ \\
\hline & Total & 25.9 & - & 6.1 & 3.0 & 4.6 & 2.6 & 8.3 & - & 1.3 \\
\hline \multirow[t]{2}{*}{ Total } & $\begin{array}{r}0-25 \\
26-50 \\
51-75 \\
76+\end{array}$ & $\begin{array}{r}41.2 \\
152.9 \\
203.7 \\
168.2 \\
\end{array}$ & $\begin{array}{r}7.6 \\
8.8 \\
9.0 \\
63.1 \\
\end{array}$ & $\begin{array}{r}8.4 \\
12.6 \\
10.1 \\
17.0 \\
\end{array}$ & $\begin{array}{r}3.4 \\
10.6 \\
11.0 \\
14.1 \\
\end{array}$ & $\begin{array}{r}7.9 \\
38.8 \\
52.1 \\
26.0 \\
\end{array}$ & $\begin{array}{r}1.4 \\
42.0 \\
78.7 \\
24.2 \\
\end{array}$ & $\begin{array}{r}7.3 \\
28.7 \\
28.2 \\
17.6 \\
\end{array}$ & $\begin{array}{r}2.6 \\
10.1 \\
8.8 \\
4.0 \\
\end{array}$ & $\begin{array}{l}2.6 \\
1.3 \\
5.8 \\
2.1 \\
\end{array}$ \\
\hline & Total & 566.0 & 88.6 & 48.2 & 39.1 & 124.8 & 146.3 & 81.7 & 25.6 & 11.7 \\
\hline
\end{tabular}

$1 /$ Table may not add to totals tue to rounding. 
Table 18.--Area of aspen type commercial forest land by site productivity class, potential achieved, and physiographic class, for growing-stock trees, based on STEMS, Central Hardwood Unit, Minnesota, 1977-1/

\begin{tabular}{|c|c|c|c|c|c|c|c|}
\hline \multirow{2}{*}{$\begin{array}{l}\text { Site } \\
\text { productivity } \\
\left(\mathrm{ft}^{3} / \mathrm{ac} / \mathrm{yr}\right)\end{array}$} & \multirow{2}{*}{$\begin{array}{l}\text { Potential } \\
\text { achieved }\end{array}$} & \multirow{2}{*}{$\begin{array}{c}\text { All } \\
\text { classes } \\
\end{array}$} & \multicolumn{5}{|c|}{ Physiographic class } \\
\hline & & & Xeric & Xeromesic & Mesic & Hydromesic & Hydric \\
\hline \multirow{3}{*}{$101-120$} & Percent & $-\cdots-1-$ & $\ldots$ & ..... Thou & d acres & 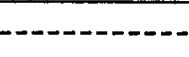 & $-0-0-0-1-1$ \\
\hline & $\begin{array}{r}0-25 \\
26-50 \\
51-75 \\
76+\end{array}$ & $\begin{array}{l}-- \\
-- \\
-- \\
-- \\
\end{array}$ & $\begin{array}{l}-- \\
-- \\
-- \\
--\end{array}$ & $\begin{array}{l}-- \\
- \\
-- \\
-- \\
\end{array}$ & $\begin{array}{l}-- \\
-- \\
-- \\
-- \\
\end{array}$ & $\begin{array}{l}-- \\
-- \\
-- \\
-- \\
\end{array}$ & $\begin{array}{l}-- \\
-- \\
-- \\
-- \\
\end{array}$ \\
\hline & Total & -- & -- & -- & -- & -- & -- \\
\hline \multirow[t]{2}{*}{$81-100$} & $\begin{array}{r}0-25 \\
26-50 \\
51-75 \\
76+\end{array}$ & $\begin{array}{l}1.3 \\
4.6 \\
2.8 \\
1.6 \\
\end{array}$ & $\begin{array}{l}-- \\
-- \\
-- \\
-\end{array}$ & $\begin{array}{l}-- \\
-- \\
--\end{array}$ & $\begin{array}{l}--\overline{3} \\
2.0 \\
1.6\end{array}$ & $\begin{array}{r}1.3 \\
1.6 \\
-- \\
-- \\
\end{array}$ & $\begin{array}{l}-- \\
-- \\
-- \\
--\end{array}$ \\
\hline & Total & 10.3 & -- & -- & 7.4 & 2.9 & -- \\
\hline \multirow[t]{2}{*}{$61-80$} & $\begin{array}{r}0-25 \\
26-50 \\
51-75 \\
76+\end{array}$ & $\begin{array}{r}17.1 \\
111.5 \\
54.3 \\
41.5 \\
\end{array}$ & $\begin{array}{r}-- \\
-- \\
1.4 \\
-- \\
\end{array}$ & $\begin{array}{l}-- \\
-- \\
-- \\
--\end{array}$ & $\begin{array}{r}11.4 \\
104.6 \\
50.0 \\
38.3 \\
\end{array}$ & $\begin{array}{l}5.7 \\
7.0 \\
3.0 \\
3.2 \\
\end{array}$ & $\begin{array}{l}-- \\
-- \\
-- \\
--\end{array}$ \\
\hline & Total & 224.5 & 1.4 & -- & 204.3 & 18.8 & - \\
\hline \multirow[t]{2}{*}{$41-60$} & $\begin{array}{r}0-25 \\
26-50 \\
51-75 \\
76+\end{array}$ & $\begin{array}{r}61.0 \\
130.2 \\
72.1 \\
42.1 \\
\end{array}$ & $\begin{array}{r}-- \\
- \\
1.4 \\
--\end{array}$ & $\begin{array}{l}-- \\
1.6 \\
-- \\
1.7\end{array}$ & $\begin{array}{r}50.6 \\
111.7 \\
57.7 \\
33.6\end{array}$ & $\begin{array}{r}10.4 \\
15.4 \\
13.0 \\
6.8 \\
\end{array}$ & $\begin{array}{l}-- \\
1.6 \\
-- \\
--\end{array}$ \\
\hline & Total & 305.4 & 1.4 & 3.2 & 253.7 & 45.5 & 1.6 \\
\hline \multirow[t]{2}{*}{$21-40$} & $\begin{array}{r}0-25 \\
26-50 \\
51-75 \\
76+\end{array}$ & $\begin{array}{l}9.4 \\
8.0 \\
6.9 \\
1.6 \\
\end{array}$ & $\begin{array}{l}-- \\
-- \\
-- \\
--\end{array}$ & $\begin{array}{l}-- \\
-- \\
-- \\
-\end{array}$ & $\begin{array}{l}3.0 \\
5.7 \\
5.3 \\
1.6 \\
\end{array}$ & $\begin{array}{r}6.4 \\
2.3 \\
1.6 \\
-. \\
\end{array}$ & $\begin{array}{l}-- \\
-- \\
-- \\
--\end{array}$ \\
\hline & Total & 25.9 & -- & $\cdots$ & 15.6 & 10.3 & -- \\
\hline \multirow[t]{2}{*}{ Total } & $\begin{array}{r}0-25 \\
26-50 \\
51-75 \\
76+\end{array}$ & $\begin{array}{r}88.7 \\
254.3 \\
136.1 \\
86.9 \\
\end{array}$ & $\begin{array}{r}-- \\
-- \\
2.7 \\
--\end{array}$ & $\begin{array}{l}-\overline{6} \\
1.6 \\
-- \\
1.7\end{array}$ & $\begin{array}{r}65.0 \\
224.9 \\
115.8 \\
75.1 \\
\end{array}$ & $\begin{array}{l}23.7 \\
26.3 \\
17.5 \\
10.0 \\
\end{array}$ & $\begin{array}{r}-- \\
1.6 \\
-- \\
--\end{array}$ \\
\hline & Total & 566.0 & 2.7 & 3.2 & 480.9 & 77.5 & 1.6 \\
\hline
\end{tabular}

$1 /$ Table may not add to totals due to rounding. 
Table 19.--Area of aspen type commercial forest land by site productivity class, potential achieved, and physiographic class, for al1 live trees, based on STEMS, Central Hardwood Unit, Minnesota, 1977 1 \}

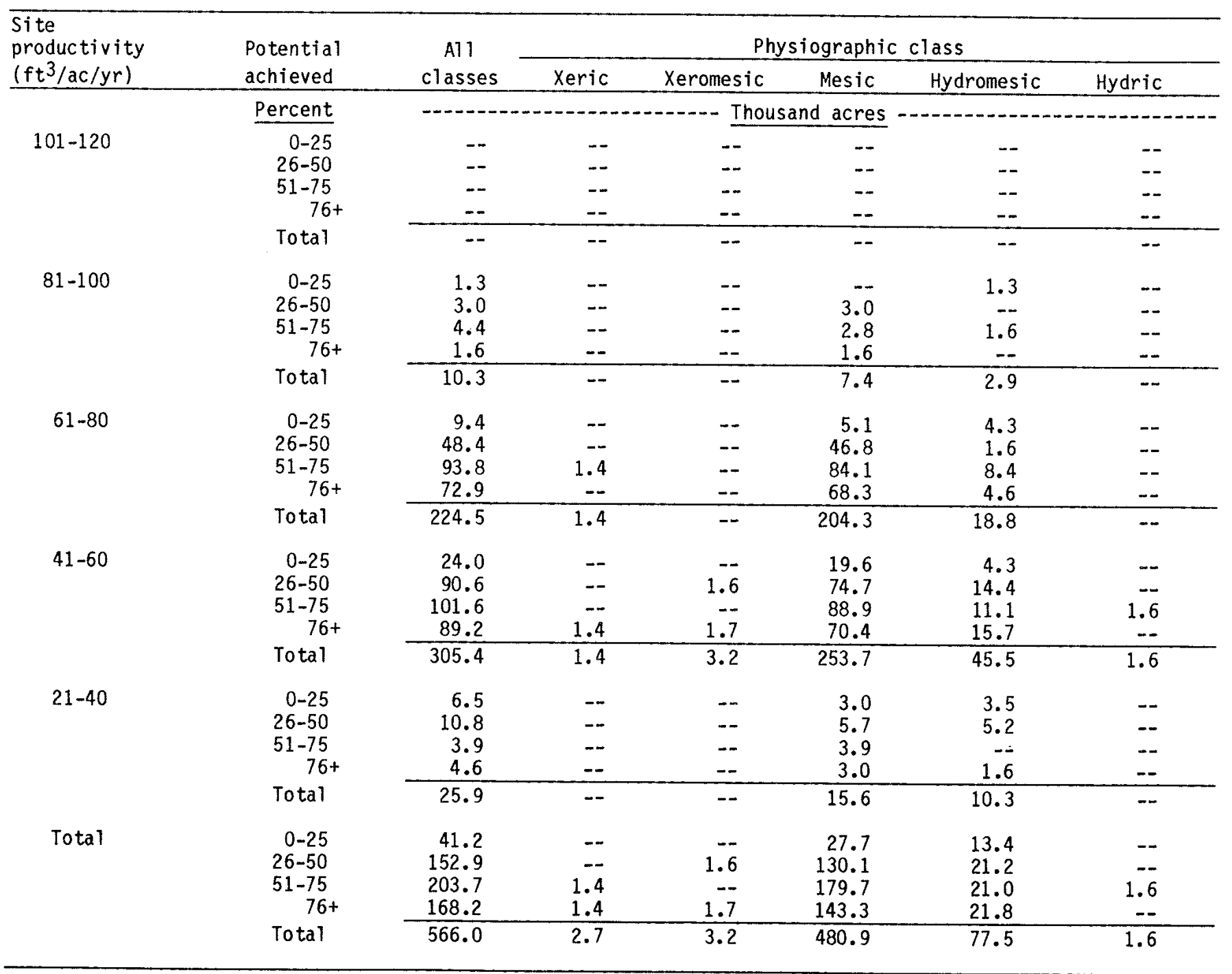

\footnotetext{
1/Table may not add to totals due to rounding.
} 
Table 20.--Area of aspen type commercial forest land by site productivity class, potential achieved, and stand-age class, for growing-stock trees, based on STEMS, Prairie Unit, Minnesota, 19771/

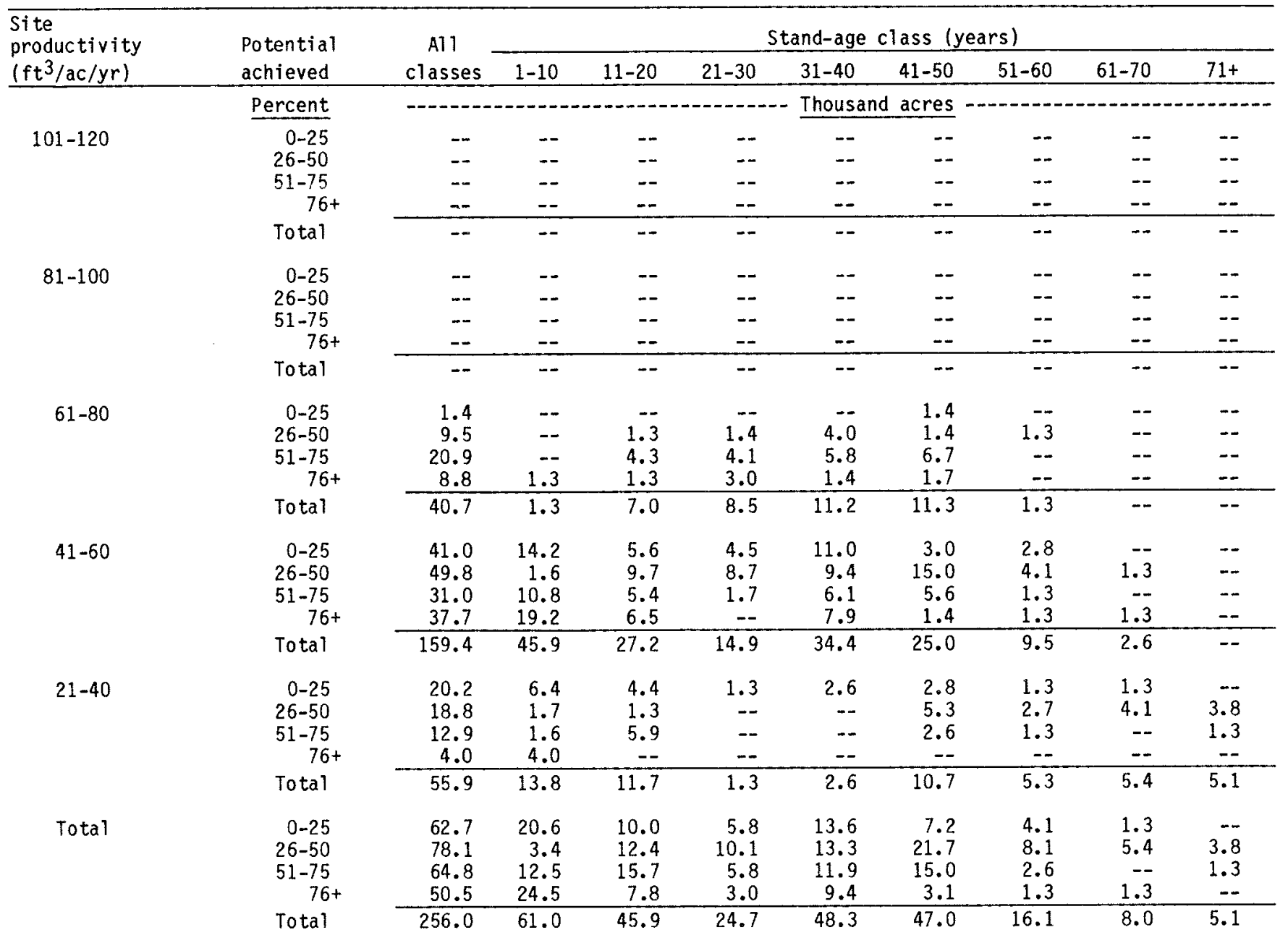

$1 /$ Table may not add to totals due to rounding. 
Table 21.--Area of aspen type commercial forest land by site productivity class, potential achieved, and stand-age class, for all live trees, based on STEMS, Prairie Unit, Minnesota, 1977피

\begin{tabular}{|c|c|c|c|c|c|c|c|c|c|c|}
\hline \multirow{2}{*}{$\begin{array}{l}\text { Site } \\
\text { productivity } \\
\left(\mathrm{ft}^{3} / \mathrm{ac} / \mathrm{yr}\right) \\
\end{array}$} & \multirow{2}{*}{$\begin{array}{l}\text { Potential } \\
\text { achieved }\end{array}$} & \multirow{2}{*}{$\begin{array}{c}\text { All } \\
\text { classes } \\
\end{array}$} & \multicolumn{8}{|c|}{ Stand-age class (years) } \\
\hline & & & $1-10$ & $11-20$ & $21-30$ & $31-40$ & $41-50$ & $51-60$ & $61-70$ & $71+$ \\
\hline \multirow{3}{*}{$101-120$} & Percent & $----\cdots$ & $-\cdots$ & $-\infty-\infty$ & $-\cdots--$ & Thousar & acres & $-\cdots-1$ & $-\cdots$ & 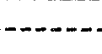 \\
\hline & $\begin{array}{r}0-25 \\
26-50 \\
51-75 \\
76+\end{array}$ & $\begin{array}{l}-- \\
-- \\
-- \\
--\end{array}$ & $\begin{array}{l}- \\
- \\
-- \\
--\end{array}$ & $\begin{array}{l}-- \\
-- \\
-- \\
-- \\
\end{array}$ & $\begin{array}{l}-- \\
-- \\
-- \\
--\end{array}$ & $\begin{array}{l}- \\
- \\
- \\
-\end{array}$ & $\begin{array}{l}-- \\
-- \\
-- \\
\end{array}$ & $\begin{array}{l}-- \\
-- \\
-- \\
--\end{array}$ & $\begin{array}{l}-- \\
-- \\
-- \\
\end{array}$ & $\begin{array}{l}-- \\
-- \\
-- \\
\end{array}$ \\
\hline & Total & -- & -- & -- & -- & -- & -- & -- & $\overline{--}$ & $\cdots$ \\
\hline \multirow[t]{2}{*}{$81-100$} & $\begin{array}{r}0-25 \\
26-50 \\
51-75 \\
76+\end{array}$ & $\begin{array}{l}-- \\
-- \\
-- \\
\end{array}$ & $\begin{array}{l}-- \\
-- \\
-- \\
\end{array}$ & $\begin{array}{l}-- \\
-- \\
-- \\
--\end{array}$ & $\begin{array}{l}-- \\
-- \\
-- \\
-\end{array}$ & $\begin{array}{l}-- \\
-- \\
-- \\
--\end{array}$ & $\begin{array}{l}-- \\
-- \\
-- \\
--\end{array}$ & $\begin{array}{l}-- \\
-- \\
-- \\
--\end{array}$ & $\begin{array}{l}-- \\
-- \\
-- \\
\end{array}$ & $\begin{array}{l}-- \\
-- \\
-- \\
-\end{array}$ \\
\hline & Total & -- & $\cdots$ & -- & -- & -- & -- & -- & -- & 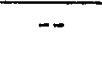 \\
\hline \multirow[t]{2}{*}{$61-80$} & $\begin{array}{r}0-25 \\
26-50 \\
51-75 \\
76+\end{array}$ & $\begin{array}{r}--\overline{2} \\
12.3 \\
25.8 \\
\end{array}$ & $\begin{array}{l}-- \\
-- \\
1.3\end{array}$ & $\begin{array}{l}-- \\
1.3 \\
3.0 \\
2.6 \\
\end{array}$ & $\begin{array}{r}-- \\
-- \\
-\overline{-5} \\
\end{array}$ & $\begin{array}{l}--- \\
1.3 \\
5.0 \\
4.9 \\
\end{array}$ & $\begin{array}{r}-- \\
-- \\
2.9 \\
8.4\end{array}$ & $\begin{array}{l}-- \\
-- \\
1.3 \\
--\end{array}$ & $\begin{array}{l}-- \\
-- \\
-- \\
-\end{array}$ & $\begin{array}{l}-- \\
-- \\
--\end{array}$ \\
\hline & Total & 40.7 & 1.3 & 7.0 & 8.5 & 11.2 & 11.3 & 1.3 & -- & -- \\
\hline \multirow[t]{2}{*}{$41-60$} & $\begin{array}{r}0-25 \\
26-50 \\
51-75 \\
76+\end{array}$ & $\begin{array}{l}15.4 \\
37.2 \\
43.3 \\
63.5 \\
\end{array}$ & $\begin{array}{r}7.8 \\
3.4 \\
3.0 \\
31.6 \\
\end{array}$ & $\begin{array}{r}1.1 \\
2.7 \\
8.9 \\
14.5 \\
\end{array}$ & $\begin{array}{r}1.7 \\
10.4 \\
2.8 \\
-- \\
\end{array}$ & $\begin{array}{r}4.8 \\
5.7 \\
14.6 \\
9.3 \\
\end{array}$ & $\begin{array}{r}-- \\
12.3 \\
8.5 \\
4.2 \\
\end{array}$ & $\begin{array}{l}-\overline{-} \\
4.8 \\
2.1 \\
\end{array}$ & $\begin{array}{l}-- \\
-- \\
1.3 \\
1.3 \\
\end{array}$ & $\begin{array}{l}-- \\
-- \\
-- \\
--\end{array}$ \\
\hline & Total & 159.4 & 45.9 & 27.2 & 14.9 & 34.4 & 25.0 & 9.5 & 2.6 & -- \\
\hline \multirow[t]{2}{*}{$21-40$} & $\begin{array}{r}0-25 \\
26-50 \\
51-75 \\
76+\end{array}$ & $\begin{array}{r}4.4 \\
22.0 \\
16.1 \\
13.4 \\
\end{array}$ & $\begin{array}{l}3.0 \\
1.7 \\
1.6 \\
7.4 \\
\end{array}$ & $\begin{array}{r}-- \\
4.4 \\
1.3 \\
5.9 \\
\end{array}$ & $\begin{array}{r}-- \\
1.3 \\
-- \\
- \\
\end{array}$ & $\begin{array}{r}-- \\
2.6 \\
-- \\
--\end{array}$ & $\begin{array}{r}1.4 \\
5.3 \\
4.0 \\
-- \\
\end{array}$ & $\begin{array}{r}--- \\
2.7 \\
2.7 \\
-- \\
\end{array}$ & $\begin{array}{r}-- \\
4.0 \\
1.4 \\
-- \\
\end{array}$ & $\begin{array}{r}-- \\
-- \\
5.1 \\
-- \\
\end{array}$ \\
\hline & Total & 55.9 & 13.8 & 11.7 & 1.3 & 2.6 & 10.7 & 5.3 & 5.4 & 5.1 \\
\hline \multirow[t]{2}{*}{ Total } & $\begin{array}{r}0-25 \\
26-50 \\
51-75 \\
76+\end{array}$ & $\begin{array}{r}19.8 \\
61.9 \\
71.6 \\
102.7 \\
\end{array}$ & $\begin{array}{r}10.8 \\
5.1 \\
4.7 \\
40.4 \\
\end{array}$ & $\begin{array}{r}1.1 \\
8.4 \\
13.3 \\
23.1 \\
\end{array}$ & $\begin{array}{r}1.7 \\
11.7 \\
2.8 \\
8.5 \\
\end{array}$ & $\begin{array}{r}4.8 \\
9.7 \\
19.7 \\
14.1 \\
\end{array}$ & $\begin{array}{l}1.4 \\
17.6 \\
15.3 \\
12.6 \\
\end{array}$ & $\begin{array}{r}-- \\
5.4 \\
8.1 \\
2.6 \\
\end{array}$ & $\begin{array}{l}-- \\
4.0 \\
2.8 \\
1.3 \\
\end{array}$ & $\begin{array}{r}-- \\
-- \\
5.1 \\
-- \\
\end{array}$ \\
\hline & Total & 256.0 & 61.0 & 45.9 & 24.7 & 48.3 & 47.0 & 16.1 & 8.0 & 5.1 \\
\hline
\end{tabular}

$1 /$ Table may not add to totals due to rounding. 
Table 22.--Area of aspen type commercial forest 1 and by site productivity class, and potential achieved, and physiographic class, for growing-stock trees, based on STEMS, Prairie Unit, Minnesota, 1977 $1 /$

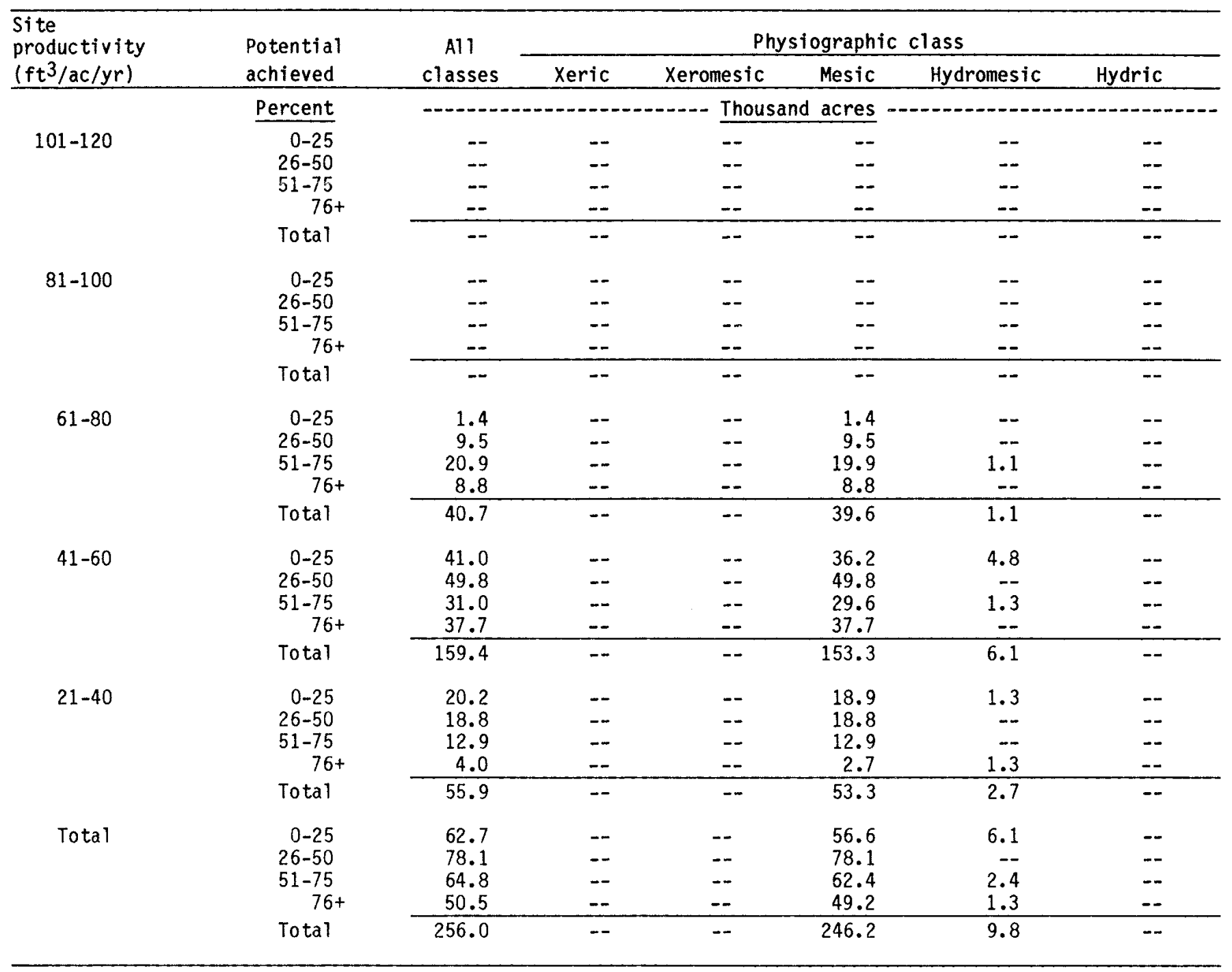

1/Table may not add to totals due to rounding. 
Table 23.--Area of aspen type commercial forest land by site productivity class, potential achieved, and physiographic class, for all live trees, based on STEMS, Prairie Unit, Minnesota, 1977 1 -

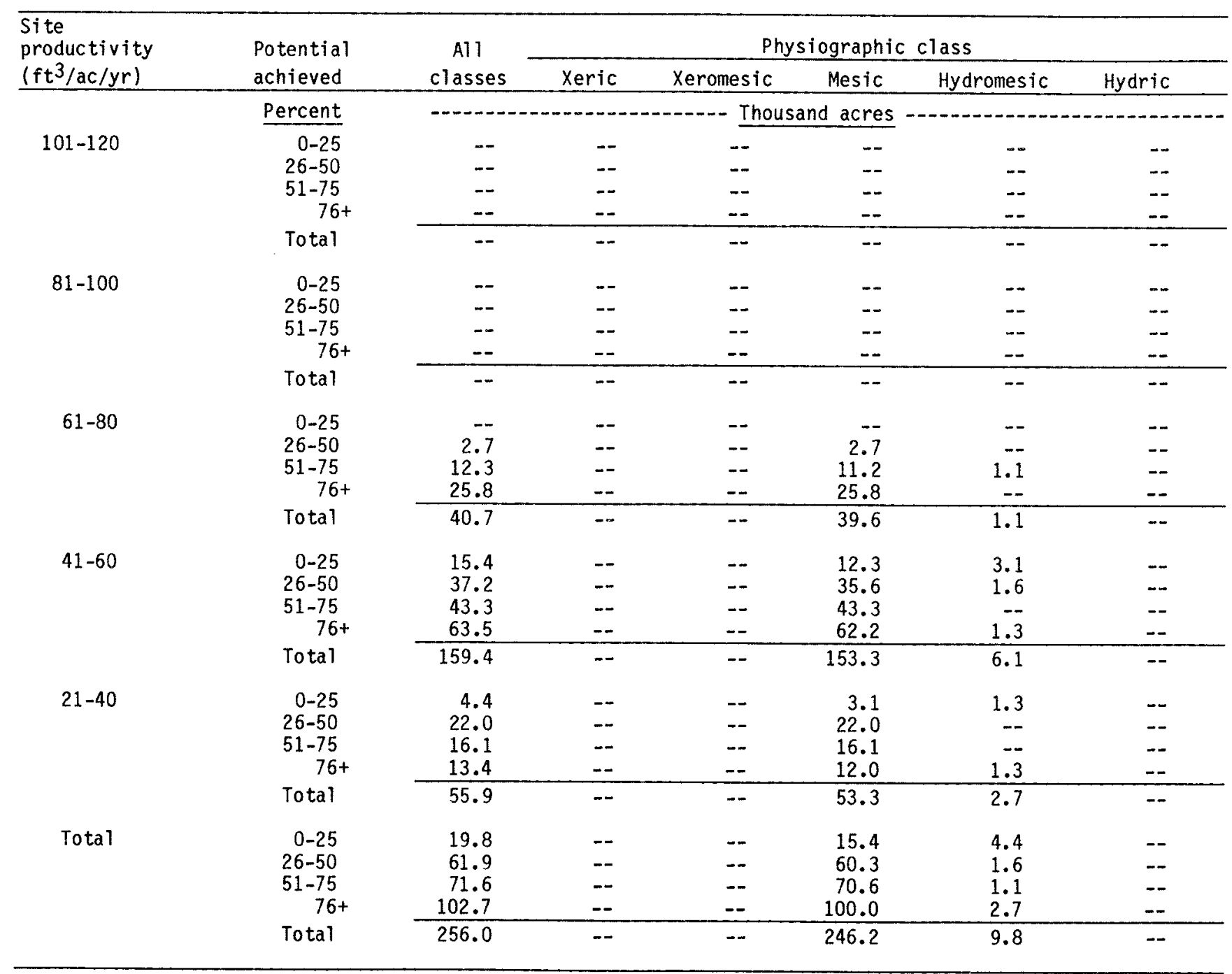

$1 /$ Table may not add to totals due to rounding. 
Table 24.--Area of aspen type commercial forest land by site productivity class, potential achieved, and stand-age class, for growing-stock trees, based on Kittredge and Gevorkiantz (1929), Minnesota, 1977 I

\begin{tabular}{|c|c|c|c|c|c|c|c|c|c|c|}
\hline \multirow{2}{*}{$\begin{array}{l}\text { Site } \\
\text { productivity } \\
\left(\mathrm{ft}^{3} / \mathrm{ac} / \mathrm{yr}\right)\end{array}$} & \multirow{2}{*}{$\begin{array}{l}\text { Potential } \\
\text { achieved }\end{array}$} & \multirow{2}{*}{$\begin{array}{c}\text { Al1 } \\
\text { classes } \\
\end{array}$} & \multicolumn{8}{|c|}{ Stand-age class (years) } \\
\hline & & & $1-10$ & $11-20$ & $21-30$ & $31-40$ & $41-50$ & $51-60$ & $61-70$ & $71+$ \\
\hline & Percent & $---\cdots$ & $-\infty$ & --- & $-\infty-\infty$ & Thousa & d acres & 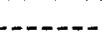 & $\ldots-\infty$ & 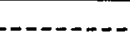 \\
\hline \multirow[t]{2}{*}{$101-120$} & $\begin{array}{r}0-25 \\
26-50 \\
51-75 \\
76+\end{array}$ & $\begin{array}{r}12.6 \\
94.6 \\
104.1 \\
55.9\end{array}$ & $\begin{array}{l}1.4 \\
2.6 \\
1.3 \\
8.5\end{array}$ & $\begin{array}{l}3.3 \\
4.1 \\
5.6 \\
4.4\end{array}$ & $\begin{array}{r}2.6 \\
11.4 \\
7.0 \\
17.6\end{array}$ & $\begin{array}{r}2.7 \\
21.0 \\
33.7 \\
10.2\end{array}$ & $\begin{array}{r}1.2 \\
26.8 \\
32.4 \\
5.6\end{array}$ & $\begin{array}{r}1.4 \\
17.8 \\
15.9 \\
5.5\end{array}$ & $\begin{array}{l}-\overline{-} \\
4.2 \\
-\overline{-} \\
1.4\end{array}$ & $\begin{array}{l}-\overline{7} \\
8.7 \\
2.7\end{array}$ \\
\hline & Total & 267.2 & 13.8 & 17.4 & 38.6 & 67.6 & 66.0 & 40.6 & 5.6 & 17.6 \\
\hline \multirow[t]{2}{*}{$81-100$} & $\begin{array}{r}0-25 \\
26-50 \\
51-75 \\
76+\end{array}$ & $\begin{array}{l}124.9 \\
689.3 \\
675.0 \\
454.2 \\
\end{array}$ & $\begin{array}{r}31.5 \\
27.5 \\
19.7 \\
114.9 \\
\end{array}$ & $\begin{array}{r}9.0 \\
39.3 \\
43.2 \\
42.5 \\
\end{array}$ & $\begin{array}{r}9.3 \\
75.4 \\
57.4 \\
36.0\end{array}$ & $\begin{array}{r}19.2 \\
158.9 \\
148.0 \\
95.9\end{array}$ & $\begin{array}{r}25.0 \\
184.3 \\
193.7 \\
104.2 \\
\end{array}$ & $\begin{array}{r}18.5 \\
142.7 \\
125.9 \\
47.3\end{array}$ & $\begin{array}{r}5.8 \\
34.0 \\
61.1 \\
8.0\end{array}$ & $\begin{array}{r}6.6 \\
27.2 \\
25.9 \\
5.3\end{array}$ \\
\hline & Total & $1,943.3$ & 193.6 & 134.0 & 178.2 & 422.0 & 507.3 & 334.4 & 108.9 & 65.0 \\
\hline \multirow[t]{2}{*}{$61-80$} & $\begin{array}{r}0-25 \\
26-50 \\
51-75 \\
76+\end{array}$ & $\begin{array}{l}182.8 \\
612.2 \\
616.1 \\
622.1 \\
\end{array}$ & $\begin{array}{r}68.0 \\
32.1 \\
53.6 \\
160.1 \\
\end{array}$ & $\begin{array}{l}28.6 \\
30.2 \\
41.3 \\
87.8 \\
\end{array}$ & $\begin{array}{l}26.4 \\
34.0 \\
51.0 \\
28.5\end{array}$ & $\begin{array}{l}14.3 \\
81.6 \\
77.0 \\
61.7 \\
\end{array}$ & $\begin{array}{r}19.8 \\
169.6 \\
152.6 \\
104.1 \\
\end{array}$ & $\begin{array}{r}12.0 \\
133.2 \\
164.6 \\
107.6\end{array}$ & $\begin{array}{r}5.4 \\
62.5 \\
53.4 \\
40.5\end{array}$ & $\begin{array}{r}8.3 \\
69.1 \\
22.7 \\
31.9\end{array}$ \\
\hline & Total & $2,033.1$ & 313.7 & 187.9 & 139.9 & 234.5 & 446.0 & 417.4 & 161.8 & 131.9 \\
\hline \multirow[t]{2}{*}{$41-60$} & $\begin{array}{r}0-25 \\
26-50 \\
51-75 \\
76+\end{array}$ & $\begin{array}{l}121.5 \\
191.1 \\
219.8 \\
279.5 \\
\end{array}$ & $\begin{array}{l}48.5 \\
26.3 \\
14.9 \\
84.0 \\
\end{array}$ & $\begin{array}{l}20.2 \\
24.4 \\
44.5 \\
52.0 \\
\end{array}$ & $\begin{array}{r}16.5 \\
14.6 \\
19.3 \\
8.4 \\
\end{array}$ & $\begin{array}{l}12.3 \\
20.5 \\
17.9 \\
25.5\end{array}$ & $\begin{array}{r}7.2 \\
32.5 \\
35.9 \\
31.5\end{array}$ & $\begin{array}{l}11.0 \\
42.9 \\
58.4 \\
31.7\end{array}$ & $\begin{array}{r}4.5 \\
18.4 \\
21.9 \\
23.4\end{array}$ & $\begin{array}{r}1.3 \\
11.6 \\
7.0 \\
22.9\end{array}$ \\
\hline & Total & 811.8 & 173.6 & 141.1 & 58.9 & 76.2 & 107.1 & 144.0 & 68.2 & 42.8 \\
\hline \multirow[t]{2}{*}{$21-40$} & $\begin{array}{r}0-25 \\
26-50 \\
51-75 \\
76+\end{array}$ & $\begin{array}{r}55.8 \\
67.3 \\
84.2 \\
114.4 \\
\end{array}$ & $\begin{array}{r}18.9 \\
10.9 \\
8.0 \\
24.1 \\
\end{array}$ & $\begin{array}{r}17.8 \\
14.1 \\
4.4 \\
29.5 \\
\end{array}$ & $\begin{array}{l}6.4 \\
5.7 \\
9.0 \\
4.2 \\
\end{array}$ & $\begin{array}{l}5.8 \\
8.6 \\
3.0 \\
7.4 \\
\end{array}$ & $\begin{array}{r}2.8 \\
5.7 \\
8.0 \\
22.6\end{array}$ & $\begin{array}{r}1.4 \\
11.3 \\
19.1 \\
11.2\end{array}$ & $\begin{array}{r}5.3 \\
21.8 \\
9.8\end{array}$ & $\begin{array}{r}2.8 \\
5.5 \\
11.0 \\
5.5 \\
\end{array}$ \\
\hline & Total & 321.7 & 61.9 & 65.8 & 25.3 & 24.8 & 39.1 & 43.0 & 37.0 & 24.8 \\
\hline \multirow[t]{2}{*}{ Total } & $\begin{array}{r}0-25 \\
26-50 \\
51-75 \\
76+\end{array}$ & $\begin{array}{r}497.5 \\
1,654.5 \\
1,699.1 \\
1,526.1\end{array}$ & $\begin{array}{r}168.2 \\
99.4 \\
97.3 \\
391.5\end{array}$ & $\begin{array}{r}78.9 \\
112.1 \\
139.1 \\
216.2\end{array}$ & $\begin{array}{r}61.1 \\
141.1 \\
143.7 \\
94.8\end{array}$ & $\begin{array}{r}54.3 \\
290.6 \\
279.6 \\
200.7\end{array}$ & $\begin{array}{r}56.0 \\
418.9 \\
422.6 \\
268.1\end{array}$ & $\begin{array}{r}44.3 \\
347.9 \\
383.8 \\
203.3\end{array}$ & $\begin{array}{r}15.7 \\
124.3 \\
158.2 \\
83.1\end{array}$ & $\begin{array}{r}19.0 \\
120.1 \\
74.7 \\
68.3\end{array}$ \\
\hline & Total & $5,377.2$ & 756.5 & 546.3 & 440.8 & 825.2 & $1,165.5$ & 979.4 & 381.4 & 282.1 \\
\hline
\end{tabular}

$1 /$ Table may not add to totals due to rounding. Includes data from 1979 and 1980 for the Superior and Chippewa National Forests, respectively. 
Table 25.--Area of aspen type commercial forest land by site productivity class, potential achieved, and stand-age class, for all live trees, based on Kittredge and Gevorkiantz (1929), Minnesota, 1977 1)

\begin{tabular}{|c|c|c|c|c|c|c|c|c|c|c|}
\hline \multirow{2}{*}{$\begin{array}{l}\text { Site } \\
\text { productivity } \\
\left(\mathrm{ft}^{3} / \mathrm{ac} / \mathrm{yr}\right)\end{array}$} & \multirow{2}{*}{$\begin{array}{l}\text { Potential } \\
\text { achieved }\end{array}$} & \multirow{2}{*}{$\begin{array}{c}\text { All } \\
\text { classes }\end{array}$} & \multicolumn{8}{|c|}{ Stand-age class (years) } \\
\hline & & & $1-10$ & $11-20$ & $21-30$ & $31-40$ & 41-50 & $51-60$ & $61-70$ & $71+$ \\
\hline & Percent & ----- & $-\ldots$ & $-\ldots$ & $---n$ & Thousa & d acres & $\ldots-\ldots$ & 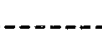 & $--\cdots-\infty$ \\
\hline \multirow[t]{2}{*}{$101-120$} & $\begin{array}{r}0-25 \\
26-50 \\
51-75 \\
76+\end{array}$ & $\begin{array}{r}7.4 \\
42.2 \\
97.5 \\
120.1 \\
\end{array}$ & $\begin{array}{r}1.4 \\
-- \\
2.7 \\
9.7 \\
\end{array}$ & $\begin{array}{l}3.3 \\
2.8 \\
2.9 \\
8.4 \\
\end{array}$ & $\begin{array}{r}1.3 \\
6.9 \\
8.5 \\
21.9 \\
\end{array}$ & $\begin{array}{l}-- \\
11.0 \\
24.7 \\
31.9\end{array}$ & $\begin{array}{l}13.2 \\
27.4 \\
25.4\end{array}$ & $\begin{array}{r}1.4 \\
2.7 \\
19.2 \\
17.3\end{array}$ & $\begin{array}{l}-- \\
2.8 \\
1.4 \\
1.4\end{array}$ & $\begin{array}{r}-- \\
2.8 \\
10.7 \\
4.1\end{array}$ \\
\hline & Total & 267.2 & 13.8 & 17.4 & 38.6 & 67.6 & 66.0 & 40.6 & 5.6 & 17.6 \\
\hline \multirow[t]{2}{*}{$81-100$} & $\begin{array}{r}0-25 \\
26-50 \\
51-75 \\
76+\end{array}$ & $\begin{array}{r}54.3 \\
364.0 \\
689.9 \\
835.2 \\
\end{array}$ & $\begin{array}{r}28.8 \\
13.1 \\
4.1 \\
147.5 \\
\end{array}$ & $\begin{array}{r}4.8 \\
27.7 \\
30.0 \\
71.5 \\
\end{array}$ & $\begin{array}{r}3.0 \\
25.3 \\
75.8 \\
74.1 \\
\end{array}$ & $\begin{array}{r}9.5 \\
89.2 \\
148.8 \\
174.5 \\
\end{array}$ & $\begin{array}{r}4.1 \\
108.6 \\
193.9 \\
200.7\end{array}$ & $\begin{array}{r}2.4 \\
76.7 \\
142.8 \\
112.5 \\
\end{array}$ & $\begin{array}{l}-- \\
12.9 \\
62.2 \\
33.8\end{array}$ & $\begin{array}{r}1.7 \\
10.4 \\
32.4 \\
20.5 \\
\end{array}$ \\
\hline & Total & $\overline{1,943.3}$ & 193.6 & 134.0 & 178.2 & 422.0 & 507.3 & 334.4 & 108.9 & 65.0 \\
\hline \multirow[t]{2}{*}{$61-80$} & $\begin{array}{r}0-25 \\
26-50 \\
51-75 \\
76+\end{array}$ & $\begin{array}{r}102.8 \\
346.3 \\
606.2 \\
977.9 \\
\end{array}$ & $\begin{array}{r}50.6 \\
18.5 \\
36.7 \\
207.9 \\
\end{array}$ & $\begin{array}{r}17.2 \\
21.6 \\
36.9 \\
112.2 \\
\end{array}$ & $\begin{array}{l}16.7 \\
30.7 \\
37.8 \\
54.8 \\
\end{array}$ & $\begin{array}{r}6.7 \\
55.9 \\
69.0 \\
102.9 \\
\end{array}$ & $\begin{array}{r}5.9 \\
87.5 \\
159.0 \\
193.6 \\
\end{array}$ & $\begin{array}{r}1.3 \\
85.4 \\
145.3 \\
185.4 \\
\end{array}$ & $\begin{array}{r}1.4 \\
32.8 \\
54.6 \\
73.0 \\
\end{array}$ & $\begin{array}{r}3.0 \\
13.8 \\
66.9 \\
48.2 \\
\end{array}$ \\
\hline & Total & $2,033.1$ & 313.7 & 187.9 & 139.9 & 234.6 & 446.0 & 417.4 & 161.8 & 131.9 \\
\hline \multirow[t]{2}{*}{$41-60$} & $\begin{array}{r}0-25 \\
26-50 \\
51-75 \\
76+\end{array}$ & $\begin{array}{r}57.6 \\
136.1 \\
183.5 \\
434.7 \\
\end{array}$ & $\begin{array}{r}34.4 \\
17.1 \\
6.1 \\
116.0 \\
\end{array}$ & $\begin{array}{r}2.6 \\
27.7 \\
27.7 \\
83.2 \\
\end{array}$ & $\begin{array}{r}5.0 \\
16.2 \\
22.3 \\
15.3\end{array}$ & $\begin{array}{r}4.4 \\
16.8 \\
20.1 \\
34.9\end{array}$ & $\begin{array}{r}2.7 \\
18.4 \\
29.3 \\
56.7\end{array}$ & $\begin{array}{r}7.0 \\
31.4 \\
50.6 \\
55.0\end{array}$ & $\begin{array}{r}1.4 \\
5.8 \\
16.7 \\
44.3\end{array}$ & $\begin{array}{r}-. \\
2.7 \\
10.8 \\
29.3\end{array}$ \\
\hline & Total & 811.8 & 173.6 & 141.1 & 58.9 & 76.2 & 107.1 & 144.0 & 68.2 & 42.8 \\
\hline \multirow[t]{2}{*}{$21-40$} & $\begin{array}{r}0-25 \\
26-50 \\
51-75 \\
76+\end{array}$ & $\begin{array}{r}24.2 \\
47.8 \\
53.2 \\
196.4 \\
\end{array}$ & $\begin{array}{r}9.7 \\
8.0 \\
3.4 \\
40.7 \\
\end{array}$ & $\begin{array}{r}7.0 \\
12.0 \\
7.2 \\
39.7 \\
\end{array}$ & $\begin{array}{r}3.2 \\
6.2 \\
5.8 \\
10.1\end{array}$ & $\begin{array}{r}4.3 \\
4.5 \\
4.5 \\
11.5\end{array}$ & $\begin{array}{r}--\overline{8} \\
5.2 \\
28.1\end{array}$ & $\begin{array}{r}7 .- \\
14.7 \\
21.1\end{array}$ & $\begin{array}{r}-. \\
1.2 \\
5.5 \\
30.3\end{array}$ & $\begin{array}{r}-. \\
2.8 \\
7.0 \\
15.0\end{array}$ \\
\hline & Total & 321.7 & 61.9 & 65.8 & 25.3 & 24.8 & 39.1 & 43.0 & 37.0 & 24.8 \\
\hline \multirow[t]{2}{*}{ Total } & $\begin{array}{r}0-25 \\
26-50 \\
51-75 \\
76+\end{array}$ & $\begin{array}{r}246.2 \\
936.4 \\
1,630.4 \\
2,564.3 \\
\end{array}$ & $\begin{array}{r}124.9 \\
56.8 \\
53.0 \\
521.9 \\
\end{array}$ & $\begin{array}{r}34.9 \\
91.8 \\
104.7 \\
314.9 \\
\end{array}$ & $\begin{array}{r}29.2 \\
85.3 \\
150.1 \\
176.2 \\
\end{array}$ & $\begin{array}{r}25.0 \\
177.4 \\
267.1 \\
355.6 \\
\end{array}$ & $\begin{array}{r}12.7 \\
233.5 \\
414.8 \\
504.5 \\
\end{array}$ & $\begin{array}{r}12.1 \\
203.5 \\
372.5 \\
391.3\end{array}$ & $\begin{array}{r}2.8 \\
55.5 \\
140.4 \\
182.8 \\
\end{array}$ & $\begin{array}{r}4.7 \\
32.5 \\
127.8 \\
117.1 \\
\end{array}$ \\
\hline & Total & $5,377.2$ & 756.5 & 546.3 & 440.8 & 825.2 & $1,165.5$ & 979.4 & 381.4 & 282.1 \\
\hline
\end{tabular}

$1 /$ Table may not add to totals due to rounding. Includes data from 1979 and 1980 for the Superior and Chippewa National Forests, respectively. 
Table 26.--Area of aspen type commercial forest land by site productivity class, potential achieved, and phsyiographic class, for growing-stock trees, based on Kittredge and Gevorkiantz, Minnesota (1929), 1977-1/

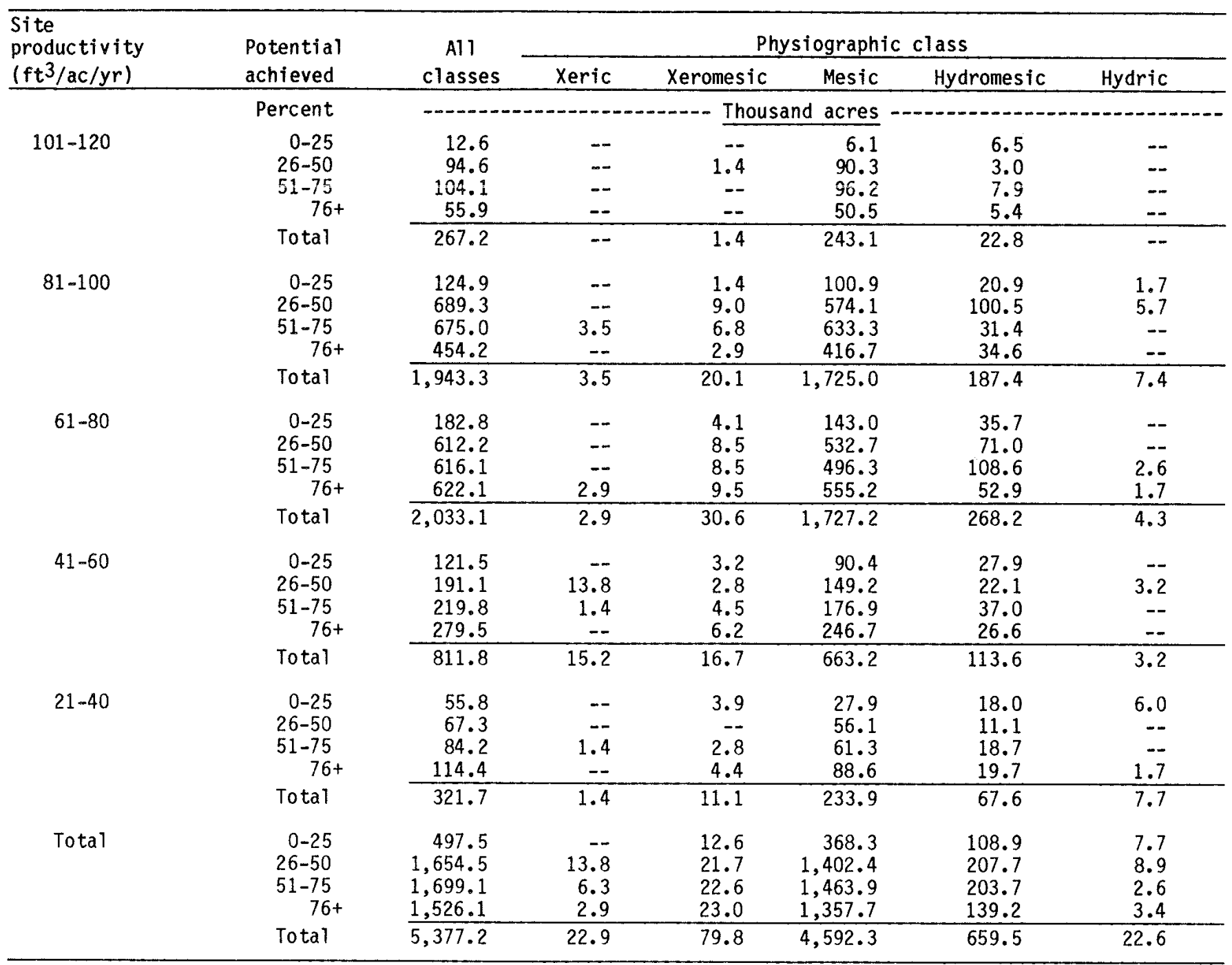

1/ Table may not add to totals due to rounding. Includes data from 1979 and 1980 for the Superior and Chippewa National Forests, respectively. 
Table 27.--Area of aspen type commercial forest 1and by site productivity class, potential achieved, and physiographic class, for all live trees, based on Kittredge and Gevorkiantz (1929), Minnesota, 1977 1 )

\begin{tabular}{|c|c|c|c|c|c|c|c|}
\hline \multirow{2}{*}{$\begin{array}{l}\text { Site } \\
\text { productivity } \\
\left(\mathrm{ft}^{3} / \mathrm{ac} / \mathrm{yr}\right)\end{array}$} & \multirow{2}{*}{$\begin{array}{l}\text { Potential } \\
\text { achieved }\end{array}$} & \multirow{2}{*}{$\begin{array}{c}\text { A11 } \\
\text { classes } \\
\end{array}$} & \multicolumn{5}{|c|}{ Physiographic class } \\
\hline & & & Xeric & Xeromesic & Mesic & Hydromesic & Hydric \\
\hline & Percent & $\cdots-\cdots$ & -non- & $\cdots-$ Thou & and acres & 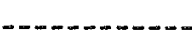 & 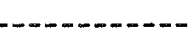 \\
\hline \multirow[t]{2}{*}{$101-120$} & $\begin{array}{r}0-25 \\
26-50 \\
51-75 \\
76+\end{array}$ & $\begin{array}{r}7.4 \\
42.2 \\
97.5 \\
120.1 \\
\end{array}$ & $\begin{array}{l}-- \\
-- \\
-- \\
--\end{array}$ & $\begin{array}{r}-- \\
1.4 \\
-- \\
-- \\
\end{array}$ & $\begin{array}{r}4.7 \\
35.6 \\
93.3 \\
109.5 \\
\end{array}$ & $\begin{array}{r}2.7 \\
5.2 \\
4.3 \\
10.6 \\
\end{array}$ & $\begin{array}{l}-- \\
-- \\
-- \\
--\end{array}$ \\
\hline & Total & 267.2 & -- & 1.4 & 243.1 & 22.8 & -- \\
\hline \multirow[t]{2}{*}{$81-100$} & $\begin{array}{r}0-25 \\
26-50 \\
51-75 \\
76+\end{array}$ & $\begin{array}{r}54.3 \\
364.0 \\
689.9 \\
835.2 \\
\end{array}$ & $\begin{array}{r}-- \\
-- \\
2.1 \\
1.4 \\
\end{array}$ & $\begin{array}{l}--- \\
7.6 \\
5.5 \\
7.0 \\
\end{array}$ & $\begin{array}{r}45.6 \\
283.7 \\
628.9 \\
766.7 \\
\end{array}$ & $\begin{array}{r}8.7 \\
68.2 \\
50.4 \\
60.1 \\
\end{array}$ & $\begin{array}{r}-- \\
4.5 \\
2.9 \\
-- \\
\end{array}$ \\
\hline & Total & $1,943.3$ & 3.5 & 20.1 & $1,725.0$ & 187.4 & 7.4 \\
\hline \multirow[t]{2}{*}{$61-80$} & $\begin{array}{r}0-25 \\
26-50 \\
51-75 \\
76+\end{array}$ & $\begin{array}{r}102.8 \\
346.3 \\
606.2 \\
977.9 \\
\end{array}$ & $\begin{array}{r}-- \\
-- \\
-- \\
2.9\end{array}$ & $\begin{array}{r}2.5 \\
4.2 \\
10.2 \\
13.7 \\
\end{array}$ & $\begin{array}{r}81.7 \\
299.1 \\
484.0 \\
862.4 \\
\end{array}$ & $\begin{array}{r}18.5 \\
43.1 \\
109.4 \\
97.3 \\
\end{array}$ & $\begin{array}{r}-- \\
-- \\
2.6 \\
1.7 \\
\end{array}$ \\
\hline & Total & $2,033.1$ & 2.9 & 30.6 & $1,727.2$ & 268.2 & 4.3 \\
\hline \multirow[t]{2}{*}{$41-60$} & $\begin{array}{r}0-25 \\
26-50 \\
51-75 \\
76+\end{array}$ & $\begin{array}{r}57.6 \\
136.1 \\
183.5 \\
434.7 \\
\end{array}$ & $\begin{array}{r}-- \\
13.8 \\
1.4 \\
-- \\
\end{array}$ & $\begin{array}{l}3.2 \\
2.8 \\
3.1 \\
7.6 \\
\end{array}$ & $\begin{array}{r}45.6 \\
94.3 \\
151.8 \\
371.5 \\
\end{array}$ & $\begin{array}{r}8.7 \\
25.2 \\
25.7 \\
54.0 \\
\end{array}$ & $\begin{array}{l}-- \\
-- \\
1.6 \\
1.6 \\
\end{array}$ \\
\hline & Total & 811.8 & 15.2 & 16.7 & 663.2 & 113.6 & 3.2 \\
\hline \multirow[t]{2}{*}{$21-40$} & $\begin{array}{r}0-25 \\
26-50 \\
51-75 \\
76+\end{array}$ & $\begin{array}{r}24.2 \\
47.8 \\
53.2 \\
196.4 \\
\end{array}$ & $\begin{array}{l}-- \\
-- \\
-\overline{1.4} \\
\end{array}$ & $\begin{array}{r}2.6 \\
-\overline{1.4} \\
7.1 \\
\end{array}$ & $\begin{array}{r}9.7 \\
35.5 \\
39.2 \\
149.5 \\
\end{array}$ & $\begin{array}{r}8.9 \\
12.3 \\
12.6 \\
33.7 \\
\end{array}$ & $\begin{array}{r}3.0 \\
-.- \\
\overline{-} \\
4.7\end{array}$ \\
\hline & Total & 321.7 & 1.4 & 11.1 & 233.9 & 67.6 & 7.7 \\
\hline \multirow[t]{2}{*}{ Total } & $\begin{array}{r}0-25 \\
26-50 \\
51-75 \\
76+\end{array}$ & $\begin{array}{r}246.2 \\
936.4 \\
1,630.4 \\
2,564.3 \\
\end{array}$ & $\begin{array}{r}-- \\
13.8 \\
3.5 \\
5.6 \\
\end{array}$ & $\begin{array}{r}8.3 \\
16.0 \\
20.2 \\
35.4 \\
\end{array}$ & $\begin{array}{r}187.3 \\
748.1 \\
1,397.2 \\
2,259.7 \\
\end{array}$ & $\begin{array}{r}47.6 \\
154.0 \\
202.3 \\
255.6 \\
\end{array}$ & $\begin{array}{l}3.0 \\
4.5 \\
7.1 \\
8.0 \\
\end{array}$ \\
\hline & Total & $5,377.2$ & 22.9 & 79.8 & $4,592.3$ & 659.5 & 22.6 \\
\hline
\end{tabular}

$1 /$ Table may not add to totals due to rounding. Includes data from 1979 and 1980 for the Superior and Chippewa National Forests, respectively. 